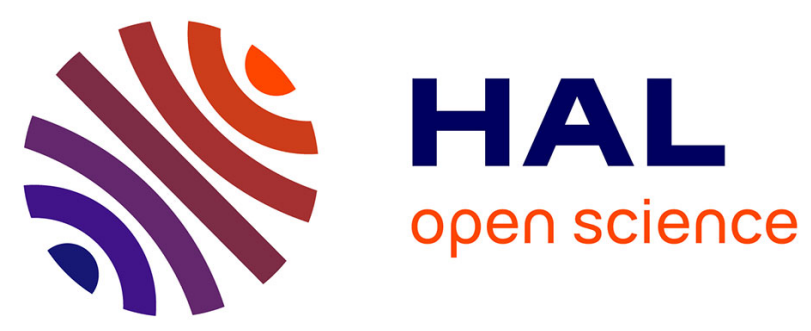

\title{
Uncertainty Quantification of Stochastic Impact Dynamic Oscillator Using a Proper Orthogonal Decomposition-Polynomial Chaos Expansion Technique
}

Biswarup Bhattacharyya, Eric Jacquelin, Denis Brizard

\section{- To cite this version:}

Biswarup Bhattacharyya, Eric Jacquelin, Denis Brizard. Uncertainty Quantification of Stochastic Impact Dynamic Oscillator Using a Proper Orthogonal Decomposition-Polynomial Chaos Expansion Technique. Journal of Vibration and Acoustics, 2020, 142 (6), 19p. 10.1115/1.4047359 . hal02971120

\author{
HAL Id: hal-02971120 \\ https://hal.science/hal-02971120
}

Submitted on 19 Oct 2020

HAL is a multi-disciplinary open access archive for the deposit and dissemination of scientific research documents, whether they are published or not. The documents may come from teaching and research institutions in France or abroad, or from public or private research centers.
L'archive ouverte pluridisciplinaire $\mathbf{H A L}$, est destinée au dépôt et à la diffusion de documents scientifiques de niveau recherche, publiés ou non, émanant des établissements d'enseignement et de recherche français ou étrangers, des laboratoires publics ou privés. 


\title{
Uncertainty quantification of stochastic impact dynamic oscillator using a proper orthogonal decomposition-polynomial chaos expansion technique
}

\author{
Biswarup Bhattacharyya* \\ Ph.D. Student \\ Univ Lyon \\ Université Claude Bernard Lyon 1 \\ IFSTTAR, LBMC UMR_T9406 \\ F69622, Lyon, France \\ Email: biswarup.bhattacharyya@ifsttar.fr
}

\author{
Eric Jacquelin \\ Professor \\ Univ Lyon \\ Université Claude Bernard Lyon 1 \\ IFSTTAR, LBMC UMR_T9406 \\ F69622, Lyon, France \\ Email: eric.jacquelin@univ-lyon1.fr
}

\author{
Denis Brizard \\ Researcher \\ Univ Lyon \\ Université Claude Bernard Lyon 1 \\ IFSTTAR, LBMC UMR_T9406 \\ F69622, Lyon, France \\ Email: denis.brizard@ifsttar.fr
}

\begin{abstract}
A proper orthogonal decomposition (POD) based polynomial chaos expansion (PCE) is utilized in this paper for the uncertainty quantification $(U Q)$ of an impact dynamic oscillator. The time-dependent non-smooth behavior and the uncertainties are decoupled using the POD approach. The uncertain response domain is reduced using the $P O D$ approach and the dominant POD modes are utilized for the UQ of the response quantity. Furthermore, the PCE model is utilized for the propagation of the input uncertainties. Two different cases of impact oscillator are considered namely single impact and multiple impact. The contact between two bodies is modeled by Hertz's law. For both the cases, UQ is performed on the projectile displacement, projectile velocity and contact force. A highly non-smooth behavior is noticed for the contact force. For that reason, most number of POD modes are required to assess UQ of contact force. All the results are compared with the Monte Carlo simulation (MCS) and time domain PCE results. Very good accuracies are observed for the PCE and the POD-PCE predicted results using much less number of model evaluations as compared to MCS. As the PCE coefficients are dependent on time, the PCE model is computed at each time-step. On the contrary, for the POD-PCE model, the PCE coefficients are computed for the number of POD modes only: it is much less than the PCE model.
\end{abstract}

\section{Introduction}

Low velocity impact dynamic behavior has been studied for beam structures [1, 2] and composite structures [3, 4]. Numerical simulation of these structures using finite element model often leads to a high computational cost and a large time consumption. For that reason, the impact dynamical systems are often modeled as a spring-mass impact oscillator [5, 1, 3] and the contact behavior is modeled using the Hertz's law [6,3]. The variation in the contact force and in the deflection can be noticed for a structure as the projectile speed varies. Due to the workmanship and the fabrication procedure, the variation

${ }^{*}$ Corresponding author 
can also be noticed in the material properties. In a nutshell, the input design parameters should be considered uncertain while designing an impact dynamic oscillator. Hence, uncertainty quantification (UQ) of the response quantities due to such uncertain input parameters is the main objective of this study.

A large number of methods have been developed in the literature for the UQ of stochastic dynamical systems [7 8 [ 9, 10]. The main difficulty with the impact dynamic oscillator is the non-smooth dynamic behavior of the response quantities [1]. Along with this, the uncertain behavior of the responses is very difficult to capture using the available methods. For the stochastic dynamical systems, polynomial chaos expansion (PCE) has gained popularity for assessing the stochastic

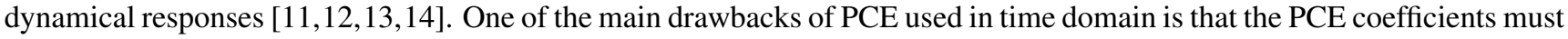
be constructed at each time-step for a dynamical systems. For that reason, PCE has been modified in several papers [7,8.9] to properly capture the time-dependent stochastic behavior. In [9], the polynomial basis is updated using the stochastic response from the previous time-step because the initial basis function is not optimal with the time. A similar procedure has been adopted in [15]. To capture the nonlinear dynamical behavior, a different approach has been adopted in [16] by rescaling the whole time domain to a small time domain and uncertainties were propagated using a principal component analysis (PCA) based PCE. The nonlinear autoregressive with exogenous input (NARX) model along with PCE has been used in [10] for the UQ of nonlinear dynamical systems. The NARX model has also been used along with the Kriging model in [17].

Although time-dependent nonlinear behavior and randomness were decoupled in the sparse NARX-PCE model [10], the NARX model requires the knowledge of the physical system. Model order reduction (MOR) techniques also decouple the time-dependent behavior and the randomness. MOR technique includes proper generalized decomposition (PGD) [18], proper orthogonal decomposition (POD, also known as PCA) [19, 20] and dynamic mode decomposition [21]. PCA based PCE has been used in [22] for reducing the dimensionality of the time-dependent stochastic diffusion problem. POD has been used in dimensionality reduction of ordinary differential equation [23] and partial differential equation [24, 20]. POD has been used in [25] for the dimensionality reduction of high-dimensional outputs. POD has the capability to decompose the time-dependent stochastic behavior using much less number of reduced bases. For that reason, POD is used in this paper in accordance with the PCE model [26] for the UQ of impact dynamic oscillators. This method has already been investigated for the smooth random dynamical systems in [27]. In this paper, the non-smooth dynamical behavior is investigated using the POD-PCE surrogate model.

This paper is organized in the following way. The PCE model and the POD approach are discussed in section 2 and section 3 respectively. The combined approach is discussed in section 4. UQ of impact dynamic oscillator is performed in section 5 and the conclusions of this study are discussed in section 6.

\section{Polynomial chaos expansion}

A brief overview of the generalized PCE model is discussed here in the context of the dynamical systems. Consider a dynamical system having $d$ independent random variables $\hat{\Xi} \in \mathbb{R}^{d}\left(\hat{\Xi}_{j}\right.$ represents the $j$-th variable). Hence, the time $(t)$ dependent response $Y(\hat{\Xi}, t)$ can be represented by the PCE model [26]:

$$
Y(\hat{\Xi}, t)=\sum_{i=1}^{\infty} y_{i}(t) \Psi^{(i)}(\hat{\Xi})
$$

where $\Psi^{(i)}(\bullet)$ are the multivariate orthogonal polynomial basis functions and $y_{i}(t)$ are the time-dependent coefficients. In the following, ' $t$ ' may indicate either the continuous time or the discretized time vector with $n_{t}$ elements. The multivariate orthogonal polynomial basis functions are constructed by the product of the univariate polynomial basis functions as follows:

$$
\Psi^{(i)}(\hat{\Xi})=\prod_{j=1}^{d} \psi^{(i, j)}\left(\hat{\Xi}_{j}\right)
$$

where $\psi^{(i, j)}\left(\hat{\Xi}_{j}\right)$ is the $i$-th univariate orthogonal polynomial basis function for the $j$-th input random variable $\hat{\Xi}_{j}$. The univariate orthogonal polynomial bases are directly related to the type of the distributions of the input random variables $\hat{\Xi}_{j}[26]$ e.g. Legendre polynomial is used for an uniformly distributed random variable and Hermite polynomial is used for a normally distributed random variable. The multivariate orthogonal polynomial bases satisfy the following condition of orthogonality:

$$
\left\langle\Psi^{(i)} \Psi^{(j)}\right\rangle=\int_{\mathbb{R}_{\hat{\underline{\Xi}}}} \Psi^{(i)}(\hat{\Xi}) \Psi^{(j)}(\hat{\Xi}) f_{\hat{\Xi}}(\hat{\Xi}) d \hat{\Xi}=h^{2} \delta_{i j}
$$


where $\delta$ is the Kronecker delta and $h$ is a constant which is 1 for the orthonormal polynomial basis function. $f_{\hat{\underline{\Xi}}}(\hat{\Xi})$ is the joint probability distribution function (PDF) of the input random variables.

For the practical implementation of the PCE model, the expression in Equation 1 is truncated with some finite number of terms in the polynomial bases. Hence, the truncated PCE is represented by:

$$
Y(\hat{\Xi}, t) \approx \sum_{i=1}^{n} y_{i}(t) \Psi^{(i)}(\hat{\Xi})
$$

where $n$ is the finite number of polynomial bases in the PCE model which can be found as follows:

$$
n=\left(\begin{array}{c}
d+P \\
P
\end{array}\right)=\frac{(d+P) !}{d ! P !}
$$

where ' $P$ ' is the maximum degree of the multivariate orthogonal polynomial bases.

PCE is utilized in this paper in a non-intrusive way. For the non-intrusive approach, initially the responses are computed as though they were deterministic at some predefined sample points (experimental design points). Therefore, the samples of the input random variables $\hat{\Xi}$ is given by $\Xi \in \mathbb{R}^{N \times d}$ matrix ( $N$ is the number of sample points) and the truncated PCE model can be identified from:

$$
Y(\Xi, t) \approx \sum_{i=1}^{n} \Psi^{(i)}(\Xi) y_{i}(t)
$$

In Equation 6 $Y=\left\{Y_{1}, Y_{2}, \ldots, Y_{N}\right\}^{T} \in \mathbb{R}^{N \times n_{t}}$ is a matrix having the responses at the initial $N$ samples and $n_{t}$ is the total number of discretized points in the time domain. $\Xi$ is a matrix having $N$ samples of the $d$-dimensional input random variables $\left(\Xi_{i} \in \mathbb{R}^{1 \times d}\right.$ is the $i$-th sample of the $d$ random variables). $\Psi(\Xi) \in \mathbb{R}^{N \times n}$ is the multivariate orthogonal polynomial basis matrix: element $(i, j)$ is $\Psi_{i, j}(\Xi)=\Psi_{j}\left(\Xi_{i}\right) . y(t)=\left\{y_{1}(t), y_{2}(t), \ldots, y_{n}(t)\right\}^{T} \in \mathbb{R}^{n \times n_{t}}$ is the time-dependent coefficient matrix of the PCE.

One of the most important tasks for the PCE model is the computation of the PCE coefficients. The usual way for computing the PCE coefficients is the ordinary least square (OLS) approach [28]. The PCE coefficients can be computed using the OLS approach as:

$$
\forall t_{k}, k \in\left\{1, \ldots, n_{t}\right\} ; \quad y\left(t_{k}\right)=\left(\Psi(\Xi)^{T} \Psi(\Xi)\right)^{-1} \Psi(\Xi)^{T} Y\left(\Xi, t_{k}\right)
$$

Hence, for an impact dynamic oscillator, the coefficients must be computed at $n_{t}$ number of time-steps which is computationally expensive. For that reason, POD is used in this paper to decouple the time domain and the randomness.

\section{Proper orthogonal decomposition}

Proper orthogonal decomposition is mainly used for the identification of low order bases for a given set of snapshots [29]. The orthogonal bases are selected corresponding to the dominating singular values of the snapshots. In our case, we have a similar problem of time-dependent stochastic response quantities. Recall the responses of a dynamical system for $N$ initial samples $Y(\Xi, t) \in \mathbb{R}^{N \times n_{t}}$ : each column of matrix $Y$ contains the stochastic response at each time-step. Hence, a kind of correlation matrix for the stochastic response quantity [30] can be defined as:

$$
C=Y^{T} Y
$$

where the superscript ' $T$ ' denotes the transpose of the preceding matrix. The orthogonal bases are found from the eigenvalue decomposition of the correlation matrix $C$ which should satisfy the following condition:

$$
C \Phi_{i}=\lambda_{i} \Phi_{i} ; \quad i=1, \ldots, n_{t}
$$

where $\Phi_{i}$ are the orthogonal bases of the POD, which are also called POD modes. $\lambda_{i}$ are the eigenvalues of the decomposition and represent a kind of energy [19] related to the corresponding POD orthogonal modes. The POD modes are sorted 
according to the decreasing magnitude of the corresponding eigenvalues $\lambda_{i}$. The POD modes are normalized here as follows:

$$
\phi_{i}=\frac{\Phi_{i}}{\left\|\Phi_{i}\right\|}
$$

where $\|\bullet\|$ is the 2-norm of the corresponding vector. The orthogonal POD modes can also be computed using the singular value decomposition (SVD) [19].

Having the orthonormal POD modes, the uncertain response series can be represented by:

$$
Y(\hat{\Xi}, t)=\sum_{i=1}^{n_{t}} b_{i}(\hat{\Xi}) \phi_{i}^{T}(t)
$$

where $b_{i}(\hat{\Xi})$ are the random coefficient vectors corresponding to the POD orthogonal modes, which need to be evaluated.

It should be noted from Equation 11 that the responses are projected on $n_{t}$ number of orthogonal POD modes. However, in our case $N<n_{t}$. Hence, the total energy is recovered only in the first $N$ eigenvalues and the responses should be projected using only the first $N$ POD modes. Furthermore, it has been seen in the literature [29,31] that only $n_{b}<N$ POD modes are usually required to keep $99.99 \%$ of the POD energy. Therefore, using the reduced number of orthonormal POD modes, the random responses can be represented by:

$$
Y(\hat{\Xi}, t)=\sum_{i=1}^{n_{b}} b_{i}(\hat{\Xi}) \phi_{i}^{T}(t)
$$

\section{POD-PCE model}

The coefficients in Equation 12 are random and are estimated using the PCE model: the random coefficients can be expressed using the truncated PCE model (refer Equation 6 ) as:

$$
b_{i}(\hat{\Xi}) \approx \sum_{j=1}^{n} a_{i j} \Psi^{(j)}(\hat{\Xi})
$$

Coefficients $a_{i j}$ are identified from the known responses of the $N$ sample points

$$
Y(\Xi, t)=\sum_{i=1}^{n_{b}} b_{i}(\Xi) \phi_{i}^{T}(t)
$$

where $b_{i}(\Xi) \in \mathbb{R}^{N \times 1}$ is the $i$-th POD coefficient vector. Each coefficient $b_{i}(\Xi)$ is known and is computed by projecting the random response matrix $Y$ on the corresponding orthonormal POD mode:

$$
b_{i}(\Xi)=Y(\Xi, t) \phi_{i}(t) ; \quad i=1, \ldots, n_{b}
$$

Having the random coefficients, the coefficient vector $a_{i j}$ can be computed using the OLS approach (refer Equation 7). Once the truncated PCE coefficients are computed, the combined POD-PCE model is given by:

$$
Y(\hat{\Xi}, t) \approx \sum_{i=1}^{n_{b}} \sum_{j=1}^{n} a_{i j} \Psi^{(j)}(\hat{\Xi}) \phi_{i}(t)
$$

\section{UQ of impact dynamic oscillator}

In this section, the UQ of a 2 degree of freedom (DOF) impact dynamic oscillator is performed. The reference solution was computed by the MCS approach using Latin hypercube sample (LHS) points. The full PCE (referred as PCE in the 
following) and POD-PCE models were identified to predict the stochastic responses using much less samples than the MCS. The accuracy of the predicted result is illustrated using the mean relative error:

$$
\bar{\varepsilon}=\frac{1}{N_{\mathrm{MCS}}} \sum_{k=1}^{N_{\mathrm{MCS}}} \varepsilon_{k}
$$

where $N_{\text {MCS }}$ is the number of samples for the MCS. $\varepsilon_{k}$ is the relative error of the $k$-th sample point which was computed as [32]:

$$
\varepsilon_{k}=\frac{\sum_{i=1}^{n_{t}}\left[Y_{\mathrm{MCS}}\left(t_{i}, \Xi_{k}\right)-Y_{\text {pred }}\left(t_{i}, \Xi_{k}\right)\right]^{2}}{\sum_{i=1}^{n_{t}}\left[Y_{\mathrm{MCS}}\left(t_{i}, \Xi_{k}\right)-\bar{Y}_{\mathrm{MCS}}\left(\Xi_{k}\right)\right]^{2}}
$$

where $\bar{Y}_{\text {MCS }}\left(\Xi_{k}\right)$ is the mean of the MCS computed response series at the $k$-th sample point. $Y_{\text {pred }}$ is the predicted response quantity using a surrogate model.

\subsection{Problem definition}

The 2-DOF nonlinear impact oscillator considered here is shown in Figure 1. The structure is denoted by the mass $m_{s t}$ and the projectile is denoted by the mass $m_{p}$. The projectile is not attached to the nonlinear spring having stiffness $k_{c}$. The governing differential equation of the 2-DOF impact oscillator is given by:

$$
\begin{gathered}
m_{s t} \ddot{x}_{s t}+c_{s t} \dot{x}_{s t}+k_{s t} x_{s t}+f_{c}=0 \\
m_{p} \ddot{x}_{p}-f_{c}=0
\end{gathered}
$$

where the structure mass $m_{s t}$ is attached to the ground by a linear spring having stiffness $k_{s t}$ and by a damper with viscous damping $c_{s t} . x_{s t}$ and $x_{p}$ are the displacements of the structure and the projectile, respectively. $f_{c}$ is the contact force between the projectile and the nonlinear spring, and was modeled using the Hertz law [33, 34]. $f_{c}$ is defined as:

$$
\begin{aligned}
f_{c} & =k_{c}\left(x_{s t}-x_{p}\right)^{\frac{3}{2}} ; \\
& =0 \quad x_{s t} \geq x_{p} \\
& \quad ; x_{s t}<x_{p}
\end{aligned}
$$

The initial conditions for the impact oscillator are given by:

$$
\begin{aligned}
& x_{s t}(0)=0 \\
& \dot{x}_{s t}(0)=0 \\
& x_{p}(0)=0 \\
& \dot{x}_{p}(0)=v_{0}
\end{aligned}
$$

where $v_{0}$ is the initial velocity of the projectile mass $m_{p}$.

In this paper, $m_{p}, k_{c}$ and $v_{0}$ are considered uniformly distributed independent random variables with coefficient of variation 0.1 . The parameters of the distribution for all the random variables are given in Table 1] The structure mass $m_{s t}=60 \mathrm{~g}$ and the damping ratio $\zeta_{s t}=0.5 \%$ are considered for this oscillator.

UQ is performed considering the projectile displacement, the projectile velocity and the contact force as the response quantities separately. The behavior of the responses is highly dependent on the number of contacts between the structure and the projectile. For that reason, the number of contacts is tuned here varying $k_{s t}$. In this paper, two cases are considered: (i) single impact $\left(k_{s t}=240 \mathrm{MN} \mathrm{m}^{-1}\right)$, (ii) multiple impact $\left(k_{s t}=2.4 \mathrm{MN} \mathrm{m}^{-1}\right)$. The non-smooth dynamical behavior is expected to be higher in case of the multiple impact oscillator.

\subsection{Case 1: Single impact oscillator}

The impact oscillator as introduced in the previous section is tuned here such that the contact between the structure and the projectile occurs only once in the total time period. A high value of $k_{s t}=240 \mathrm{MN} \mathrm{m}^{-1}$ was considered. The time domain 


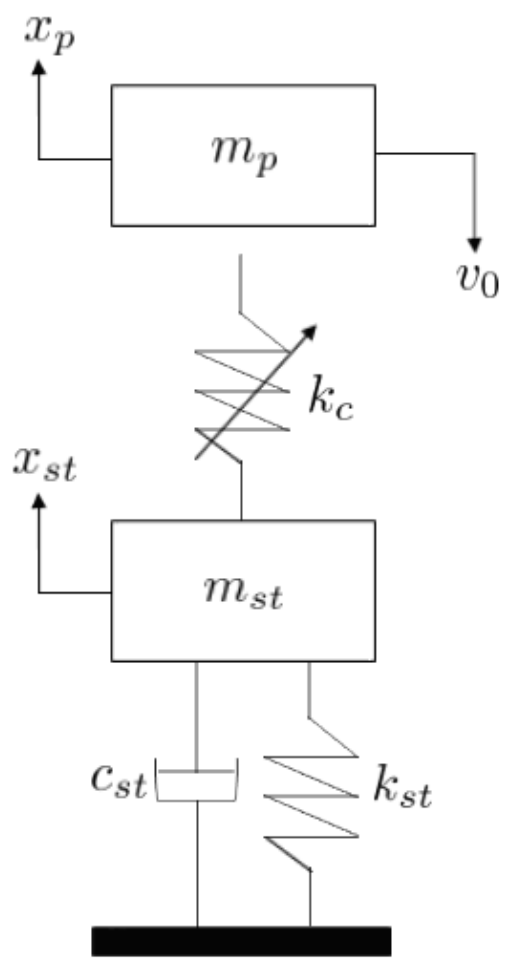

Fig. 1: 2-DOF nonlinear impact dynamic oscillator

Table 1: Parameters of the input random variables for the impact dynamic oscillator

\begin{tabular}{cccc}
\hline Random variable & Lower bound & Upper bound & Unit \\
\hline$m_{p}$ & $330-33 \sqrt{3}$ & $330+33 \sqrt{3}$ & $\mathrm{~g}$ \\
$k_{c}$ & $16 \times 10^{3}-1600 \sqrt{3}$ & $16 \times 10^{3}+1600 \sqrt{3}$ & $\mathrm{MN} \mathrm{m}^{-3 / 2}$ \\
$v_{0}$ & $-10+\sqrt{3}$ & $-10-\sqrt{3}$ & $\mathrm{~m} \mathrm{~s}^{-1}$ \\
\hline
\end{tabular}

for this system was decided as $t \in[0 \mathrm{~ms}, 1 \mathrm{~ms}]$. The time integration was performed using the MATLAB function ode 45 at a time-step of $\Delta t=1 \mu \mathrm{s}$. As a result, $n_{t}=1001$ can be found in the full time domain.

For the reference solution, the stochastic responses were computed by MCS using $N_{\mathrm{MCS}}=10^{4}$ model evaluations. On the other hand, the POD-PCE and the PCE models were constructed using much less number of model evaluations. Legendre polynomials were used for the PCE models with degree $P=2$ and $P=3$. As a result, $n=10$ and $n=20$ terms were found in the truncated PCE model for $P=2$ and $P=3$, respectively.

First of all, the influence of $N$ on the accuracy was studied for both the surrogate models. The evaluation of the mean relative errors for all the response quantities is shown in Figure 2. It is seen clearly that with the increase of $N$ the mean relative error reduces for all the response quantities. It is noticeable that for a particular polynomial degree, $\bar{\varepsilon}$ by the PCE and the POD-PCE models are almost the same with $N$. However, slightly high accuracy is noticed by the POD-PCE model for the contact force with $P=3$ and the accuracy of PCE models for the projectile velocity is greater than the POD-PCE models. An accurate identification of a model with $n$ coefficients is generally possible only if $N \geq n$. Accordingly, the responses with $P=2$ are in good agreement with the MCS responses with $N \geq 10$ because $n=10$ terms were found in the PCE polynomials, whereas $P=3$ requires at least $N=20$ model evaluation to compute the PCE coefficients accurately $(n=20)$. With $N=20$ and $P=3$, the surrogate model has almost the same accuracy as that with $P=2$ and $N=20$. Moreover, the increment of the accuracy beyond $N=20$ samples is much less for all the response quantities. Therefore, a good accuracy has achieved using $N=20$ for all responses. For that reason, all the stochastic responses are presented in the following text using $N=20$ only for the single impact oscillator.

The time-dependent mean $(\mu)$ and standard deviation $(\sigma)$ of the contact force are plotted in Figure $3 \mathrm{a}$ and Figure $3 \mathrm{~b}$, respectively. It is seen that the contact between the structure and the projectile occurs only once during the total time period. The mean and the standard deviation are computed quite well by both the surrogates using $N=20$. However, it is to be 


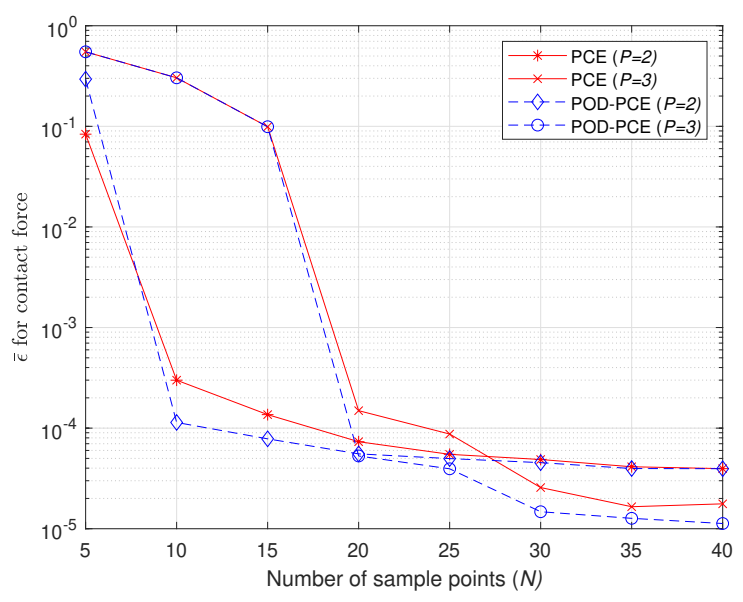

(a) $\bar{\varepsilon}$ for contact force

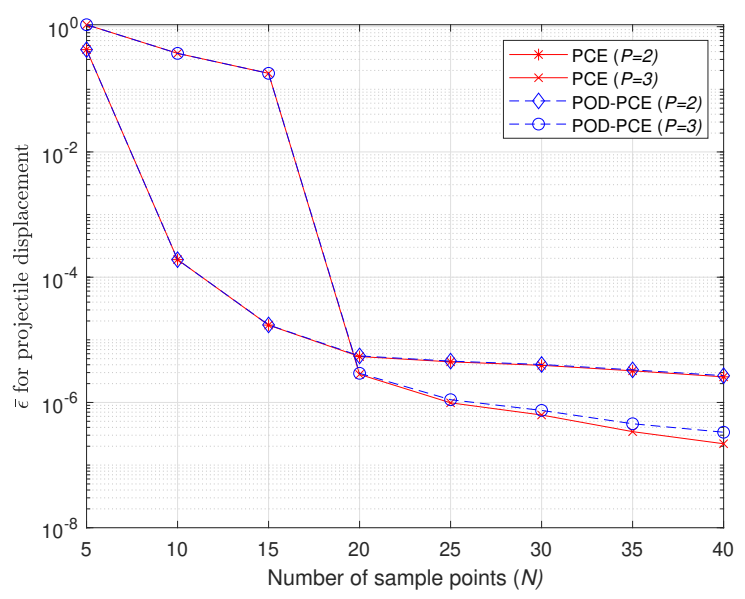

(b) $\bar{\varepsilon}$ for projectile displacement

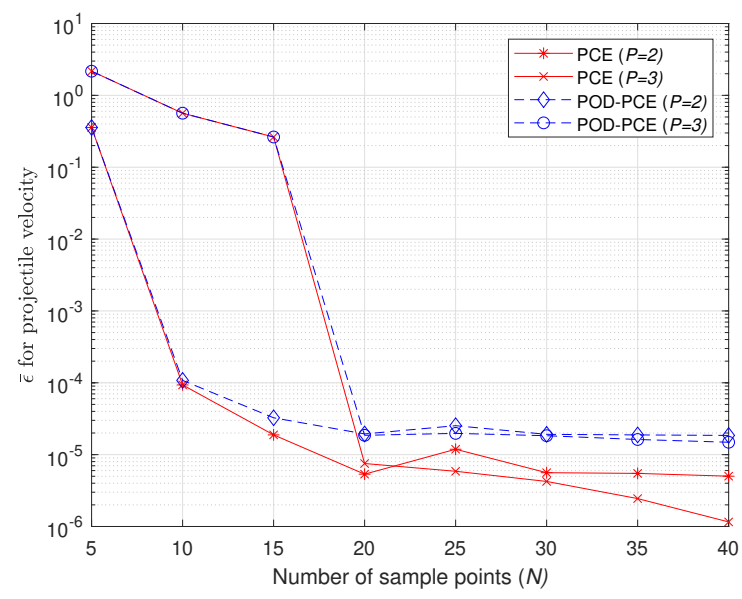

(c) $\bar{\varepsilon}$ for projectile velocity

Fig. 2: Evaluation of the mean relative errors of all the stochastic response quantities for the single impact oscillator

noted that the PCE coefficient vector was computed at each time-step for the PCE model (refer Equation 6. Conversely, the time-dependent stochastic contact force was obtained with only $n_{b}=5$ POD modes by the POD-PCE model. Hence, the PCE coefficient vector was computed only 5 times for the POD-PCE model which is much less than 1001. Indeed the total number of computed coefficients ( $n_{\text {tot }}=n \times n_{t}$ for PCE and $n_{\text {tot }}=n \times n_{b}$ for POD-PCE) is shown in Table 2 for both the surrogates, which is 200 times more for the PCE model as compared to the POD-PCE model. The time-dependent errors for the statistical moments were computed using the following expression:

$$
\varepsilon(t)=\frac{\left|\operatorname{Mom}\left(Y_{\mathrm{MCS}}\right)-\operatorname{Mom}\left(Y_{\text {pred }}\right)\right|}{\max \left|\operatorname{Mom}\left(Y_{\mathrm{MCS}}\right)\right|}
$$

where $\operatorname{Mom}(\bullet)$ is the any statistical moment $(\mu$ or $\sigma)$ of the corresponding response quantity. The time-dependent errors for $\mu_{f_{c}}$ and $\sigma_{f_{c}}$ of the contact force are shown in Figure $4 \mathrm{a}$ and Figure $4 \mathrm{~b}$, respectively: the errors are presented only during the contact and the error is zero beyond the contact. It is seen clearly that the magnitude of error in the prediction of $\sigma_{f_{c}}$ is higher than that in the prediction of $\mu_{f_{c}}$. Moreover, the POD-PCE model has better predicted $\sigma$ as compared to the PCE model using $P=3$. The overall accuracies of all the models were computed using Equation 17 and are listed in Table 2 It is noticeable that the errors predicted by the POD-PCE models are slightly less than the PCE models. Moreover, a good accuracy has been achieved by both the surrogate models using much less number of model evaluations, but the POD-PCE model requires only 5 POD modes to achieve a good accuracy.

The time-dependent statistical moments of $x_{p}$ are shown in Figure 3c and Figure 3d using $N=20$. Good agreement of surrogate predicted results with the MCS results is noticed for both the cases. As the PCE coefficients are time-dependent, the PCE coefficient vector was computed 1001 times. However, $99.99 \%$ of the energy was recovered using only 2 POD modes for 


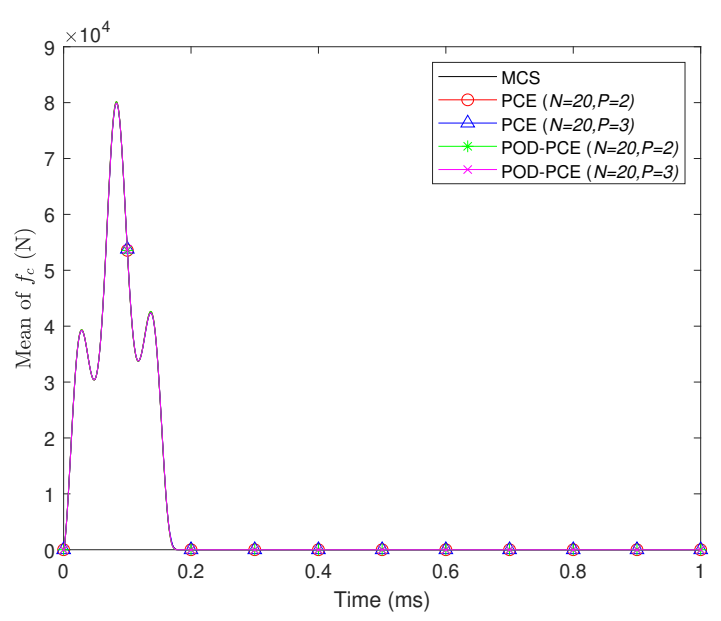

(a) Mean of $f_{c}$

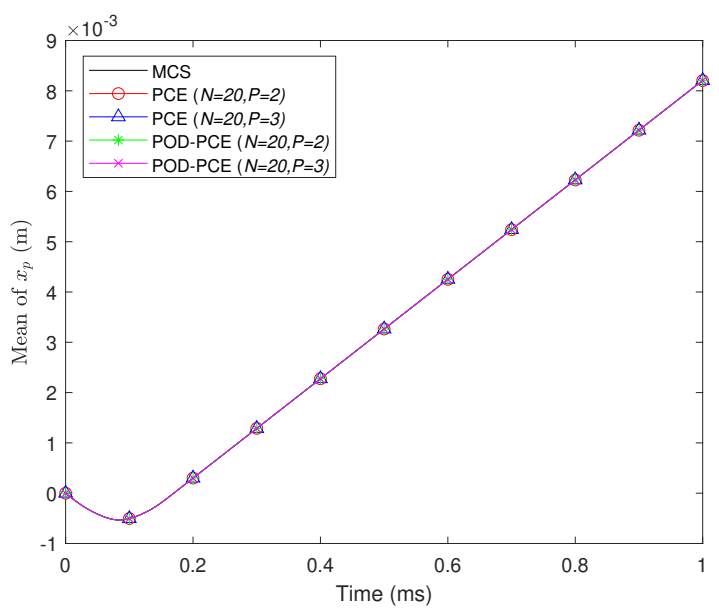

(c) Mean of $x_{p}$

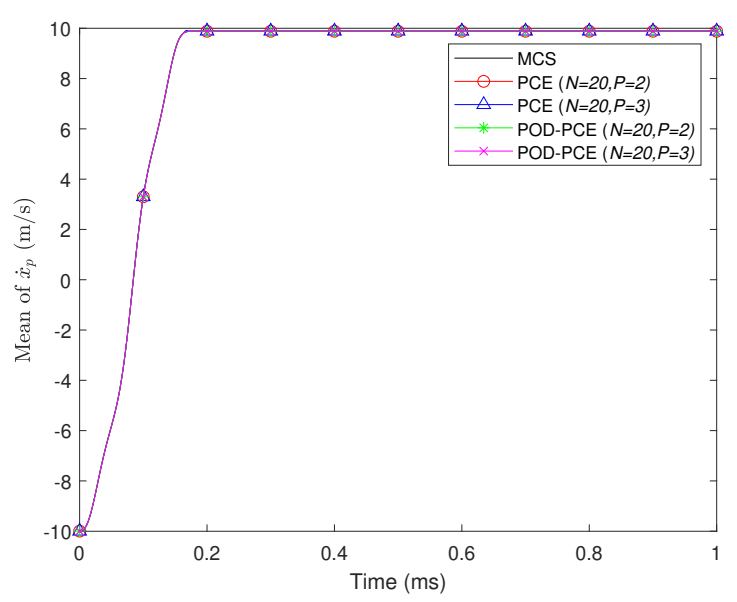

(e) Mean of $\dot{x}_{p}$

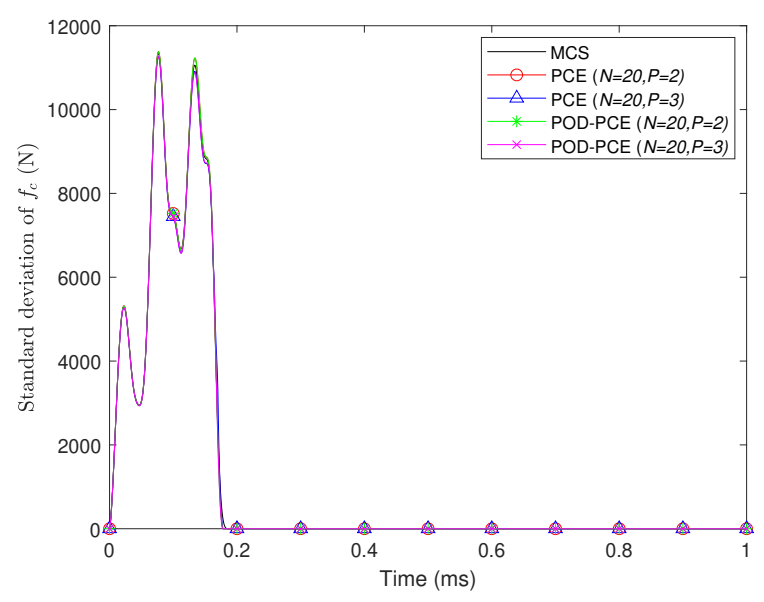

(b) Standard deviation of $f_{c}$

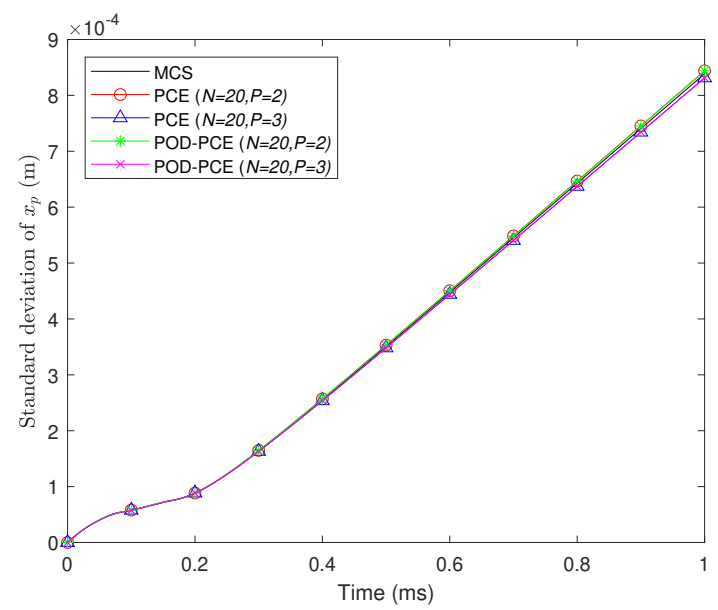

(d) Standard deviation of $x_{p}$

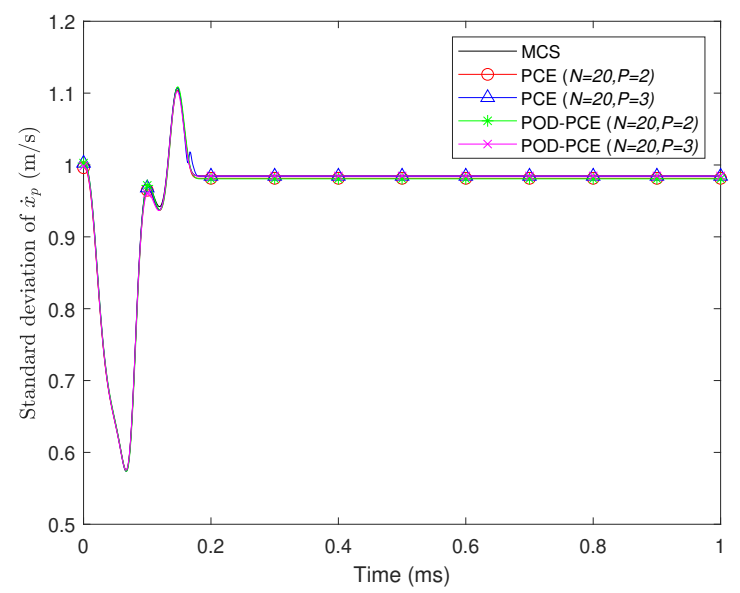

(f) Standard deviation of $\dot{x}_{p}$

Fig. 3: Time-dependent statistical moments of the stochastic response quantities for the single impact oscillator: MCS was performed using $N_{\mathrm{MCS}}=10^{4}$ model evaluations

the POD-PCE model. Hence, the PCE coefficients vector was computed only twice instead of 1001 times which ultimately lead to compute only $0.2 \%$ coefficients for the POD-PCE models with respect to the PCE models. The time-dependent errors for both the statistical moments were computed using Equation 22 and are plotted in Figure 4c and Figure 4d Although the errors are very low, lower errors were predicted using $P=3$ for both the surrogates. The overall accuracy of both the 


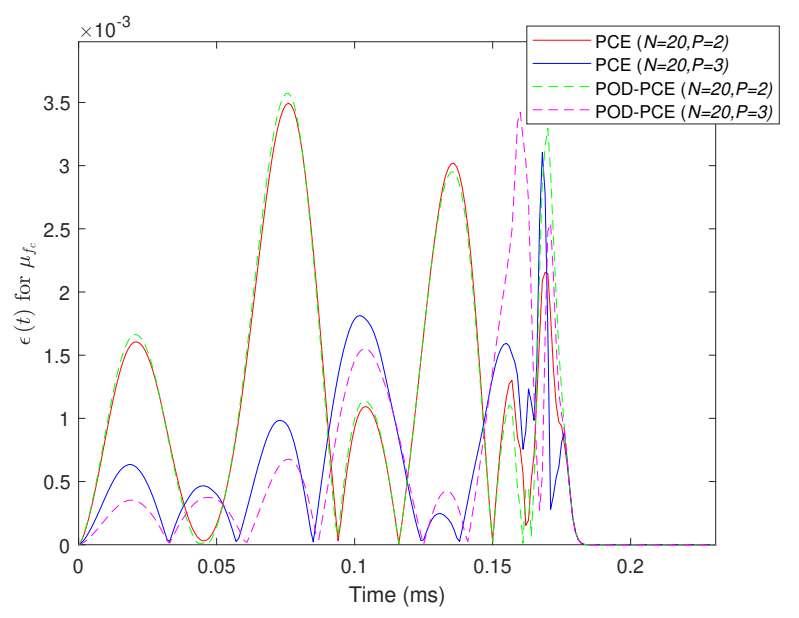

(a) Error for $\mu$ of $f_{c}$ (zoom on $\mathrm{x}$ axis)

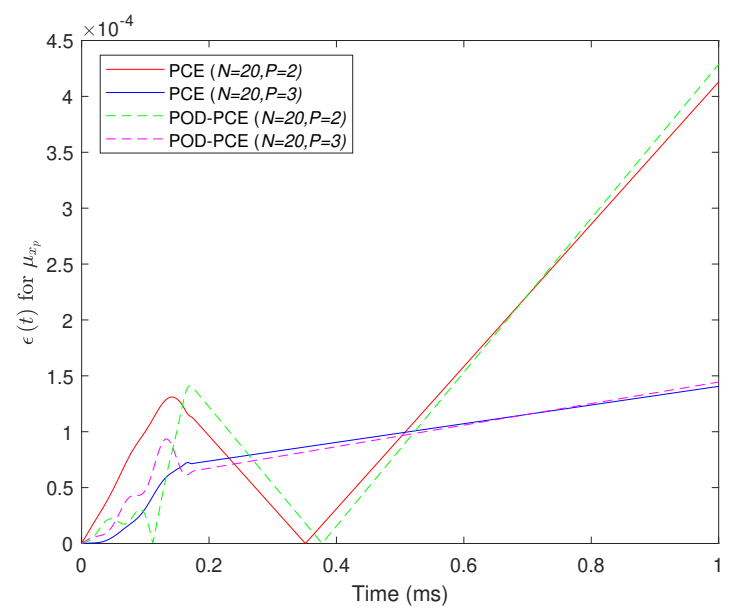

(c) Error for $\mu$ of $x_{p}$

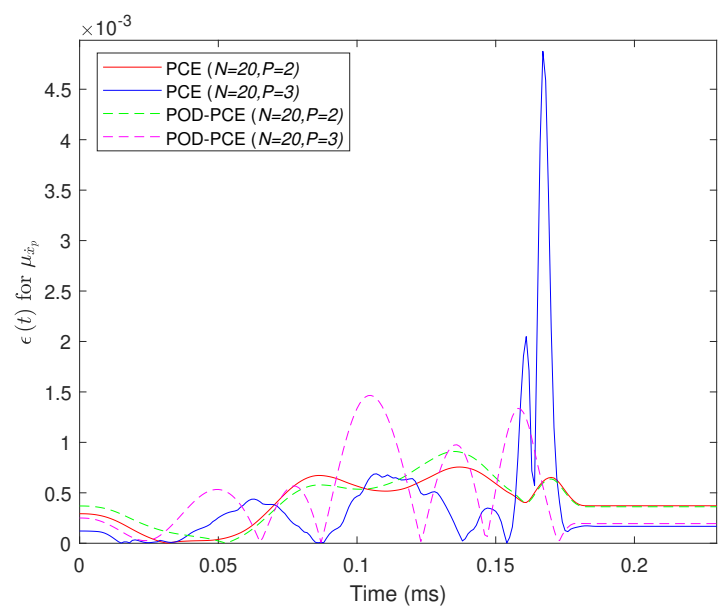

(e) Error for $\mu$ of $\dot{x}_{p}$ (zoom on $\mathrm{x}$ axis)

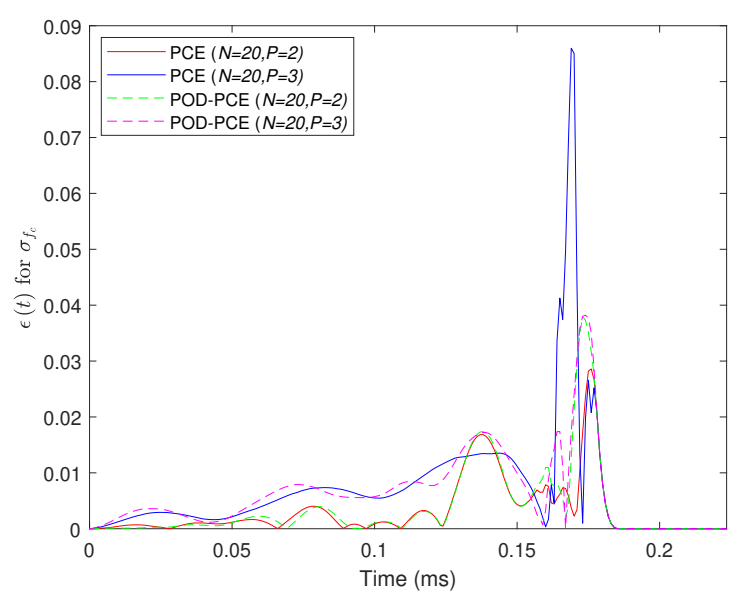

(b) Error for $\sigma$ of $f_{c}$ (zoom on $\mathrm{x}$ axis)

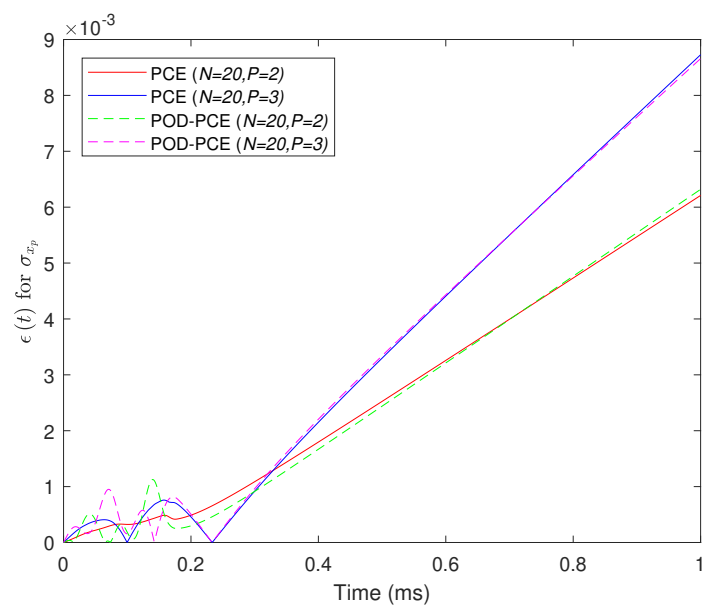

(d) Error for $\sigma$ of $x_{p}$

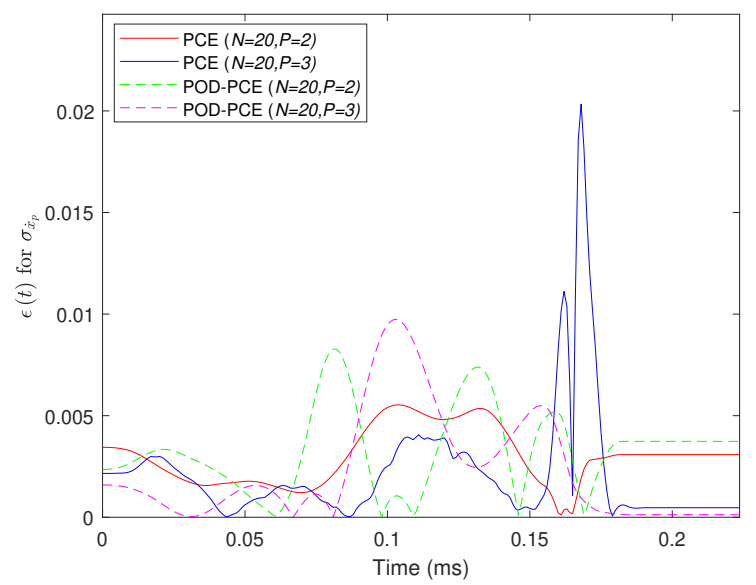

(f) Error for $\sigma$ of $\dot{x}_{p}$ (zoom on $\mathrm{x}$ axis)

Fig. 4: Time-dependent errors for the statistical moments of the stochastic responses of single impact oscillator

surrogates is measured using the mean relative error which is listed in Table 2. It is noticeable from the table that almost the same accuracies have been achieved using both the surrogates: for the POD-PCE model, it requires only 2 POD modes to achieve such a high level of accuracy.

In a similar way to the previous two responses, the stochastic projectile velocity was also computed using the surrogate models. All the parameters remain the same for the projectile velocity. Time-dependent mean and standard deviation of $\dot{x}_{p}$ 
Table 2: Accuracy of different surrogate models in assessing the stochastic response quantities for the single impact oscillator by $N=20$

\begin{tabular}{cc|ccc|ccc|ccc}
\hline & & \multicolumn{3}{|c|}{ Contact force } & \multicolumn{2}{c|}{ Projectile displacement } & \multicolumn{2}{c}{ Projectile velocity } \\
\hline Method & $P$ & $n_{b}$ & $n_{\text {tot }}$ & $\bar{\varepsilon}$ & $n_{b}$ & $n_{\text {tot }}$ & $\bar{\varepsilon}$ & $n_{b}$ & $n_{\text {tot }}$ & $\bar{\varepsilon}$ \\
\hline PCE & 2 & - & 10010 & $6.21 \times 10^{-5}$ & - & 10010 & $5.41 \times 10^{-6}$ & - & 10010 & $5.34 \times 10^{-6}$ \\
PCE & 3 & - & 20020 & $14.90 \times 10^{-5}$ & - & 20020 & $2.80 \times 10^{-6}$ & - & 20020 & $7.52 \times 10^{-6}$ \\
POD-PCE & 2 & 5 & 50 & $5.54 \times 10^{-5}$ & 2 & 20 & $5.53 \times 10^{-6}$ & 3 & 30 & $19.40 \times 10^{-6}$ \\
POD-PCE & 3 & 5 & 100 & $5.28 \times 10^{-5}$ & 2 & 40 & $2.92 \times 10^{-6}$ & 3 & 60 & $18.50 \times 10^{-6}$ \\
\hline
\end{tabular}

Table 3: Efficiency of different approaches for the single impact oscillator

\begin{tabular}{ccc|ccc}
\hline & & & Contact force & Projectile displacement & Projectile velocity \\
\hline Method & $N$ & $P$ & & CPU time (s) & \\
\hline PCE & 20 & 3 & 0.061 & 0.061 & 0.060 \\
POD-PCE & 20 & 3 & 0.003 & 0.002 & 0.002 \\
\hline MCS & $10^{4}$ & - & 41.510 & 41.510 & 41.510 \\
\hline
\end{tabular}

are shown in Figure 3e and Figure 3f, respectively. It is seen that the mean velocity is gradually increasing from negative magnitude to positive magnitude during the contact period and it remains constant after the contact. The prediction accuracies of all the surrogates are very high and are very close to each other. For the POD-PCE model, the 99.99\% energy level is achieved using only $n_{b}=3$ POD modes: indeed only 3 PCE coefficient vectors were computed for the POD-PCE model instead of 1001 PCE coefficient vectors for the PCE model.

The time-dependent errors were also computed for the predicted stochastic velocity $\dot{x}_{p}$ and are shown in Figure $4 \mathrm{e}$ and Figure 4f: the errors are plotted for the contact period only and beyond this region, the errors are constant. It is noticed that the magnitude of error is higher for $\sigma_{\dot{x}_{p}}$ as compared to $\mu_{\dot{x}_{p}}$. However, the error for $\sigma_{\dot{x}_{p}}$ by the POD-PCE model with $P=3$ is much less as compared to the others. The overall accuracies of all the surrogates are listed in Table 2 The mean relative errors by all the surrogates are close to each other.

The results obtained for 5 samples are plotted in Figure 5, as well as the results calculated from the identified surrogate models. It is seen that all the responses are predicted quite well by both the surrogate models for all the 5 samples.

The efficiency of all the approaches was measured with the number of sample points and CPU time which are listed in Table 3 The simulation was performed on Intel Core i7 CPU running at $2.90 \mathrm{GHz}$ with $32 \mathrm{~GB}$ installed memory. The CPU times presented in Table 3 were averaged for 10 simulations. The surrogate models with $P=3$ are presented only. It is seen that the number of sample points for the surrogate models is quite small as compared to the MCS approach. Further, the CPU time for the PCE model is almost 830 times smaller than the MCS approach and almost 21000 times smaller for the POD-PCE model. For the POD-PCE models, the POD was performed by the SVD approach and it depends on the minimum between the number of model evaluations $(N)$ and the number of time-steps $\left(n_{t}\right)$. For the present case, $N$ is quite small as compared to $n_{t}$. Therefore, the POD approach took approximately $0.001 \mathrm{~s}$ to obtain the POD modes. As a result, the efficiency of the POD-PCE model was almost 30 times higher than the PCE model.

\subsection{Case 2: Multiple impact oscillator}

The same oscillator as defined in section 5.1 is considered here with $k_{s t}=2.4 \mathrm{MNm}^{-1}$ such that multiple contacts occur between the structure and the projectile. The time domain considered here is $t \in[0 \mathrm{~ms}, 3 \mathrm{~ms}]$ and the time integration was performed at a time-step of $\Delta t=1 \mu \mathrm{s}$. Consequently, $n_{t}=3001$ number of time-steps can be found in the total time period.

Stochastic responses were computed considering the three input random variables as mentioned in Table 1. For the multiple impact oscillator, MCS was performed using $10^{4}$ model evaluations on LHS points for all the stochastic response quantities. In a similar way to the single impact oscillator, the influence of the number of model evaluations was investigated for the surrogate models. As this system is highly non-smooth and nonlinear in nature, initial sample for the surrogate model was decided as $N=30$. The relative error with respect to $N$ for all the response quantities is shown in Figure 6 It is noticeable that the accuracy is increased with the increase of $N$ for all the response quantities. The magnitude of error 


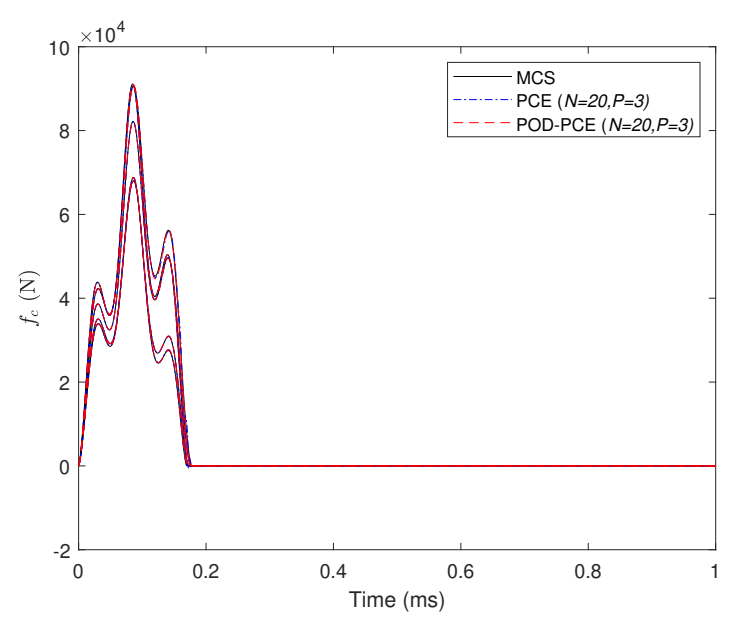

(a) Contact force

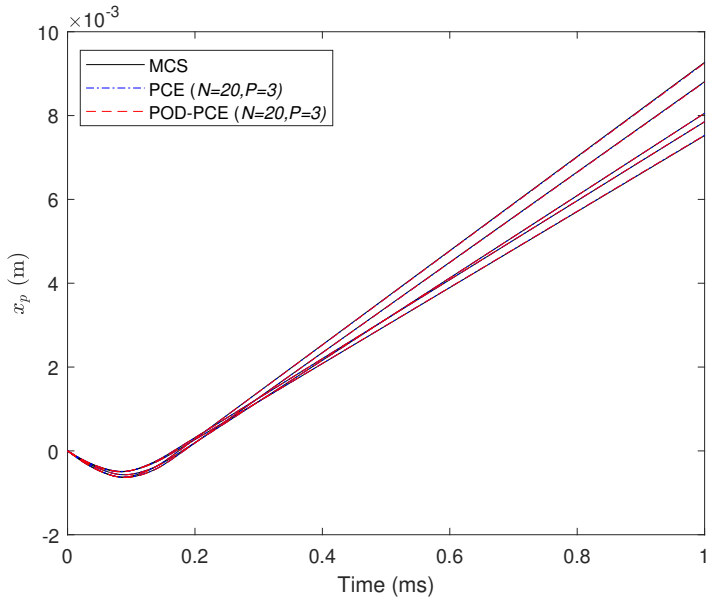

(b) Projectile displacement

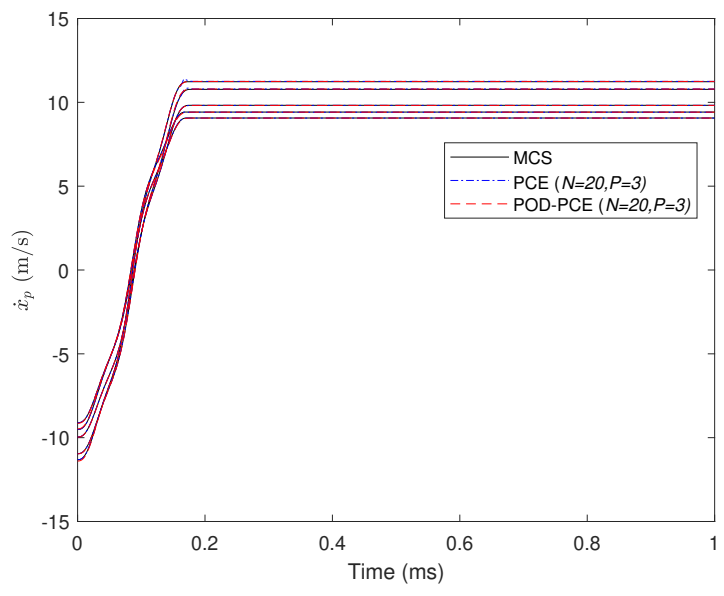

(c) Projectile velocity

Fig. 5: Comparison of responses for single impact oscillator at 5 samples: $m_{p} \in\{342.71,292.84,365.62,310.35,373.82\} \mathrm{g}$, $k_{c} \in\{13.97,13.87,14.61,14.23,13.63\} \times 10^{3} \mathrm{MNm}^{-3 / 2}, v_{0} \in-\{11.32,9.51,9.96,9.11,10.95\} \mathrm{m} \mathrm{s}^{-1}$

for the contact force is quite higher as compared to the other response quantities due to highly non-smooth and nonlinear in behavior. Moreover, a quite good accuracy has achieved using $N=50$ samples for all the response quantities. Hence, all the results are presented in following discussion using $N=50$ samples.

The stochastic behaviors of the contact force are plotted in Figure 7a and Figure 7b the results are presented magnifying on the x axis. The surrogate models were constructed using $N=50 \mathrm{LHS}$ points and predicted at $N_{\mathrm{MCS}}=10^{4}$ samples. It is seen clearly from the mean force that five contacts occur during the whole time period and the maximum contact force occurs during the second contact. Here we can see the much high non-smooth dynamic behavior of the oscillator as compared to the previous one. Despite the much high non-smooth behavior, the mean force was predicted quite well by both the surrogates. However, tiny discrepancies are noticed in the standard deviation of $f_{c}$. The time-dependent errors were also computed for both the surrogates, which are shown in Figure 8a and Figure 8b for the contact region only. The magnitude of error for $\sigma_{f_{c}}$ is high as compared to $\mu_{f_{c}}$. Both the surrogates have predicted lower error with $P=3$ as compared to $P=2$. The level of overall accuracies are listed in Table 4 It is noticeable that a large number of POD modes $\left(n_{b}=31\right)$ was required to keep $99.99 \%$ of the POD energy with the retained POD modes due to much high non-smooth nonlinear behavior: the PCE coefficient vector was therefore computed 31 times. However, it is much less than the computation of the coefficient vector for the PCE model: it is 3001 for the PCE model. Indeed $n_{\text {tot }}$ for the POD-PCE model is approximately $1 \%$ of the time-dependent PCE model.

The stochastic projectile displacement $x_{p}$ was computed by both the surrogates using 50 LHS points. Time-dependent moments of $x_{p}$ are shown in Figure 7c and Figure 7d. Both the moments are predicted quite well using both the surrogates. The time-dependent errors were computed for the moments of $x_{p}$ and are plotted in Figure 8c and Figure 8d The magnitude of error for $\sigma_{x_{p}}$ is higher as compared to $\mu_{x_{p}}$. Slightly lower errors were predicted by both the surrogates with $P=3$. Along with this, the overall accuracies of all the surrogates are listed in Table 4. It seems that both the approaches have predicted 


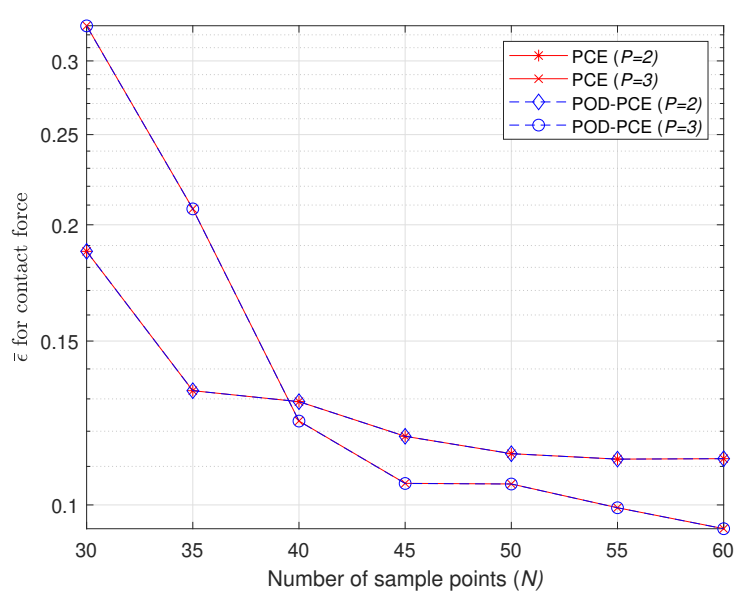

(a) $\bar{\varepsilon}$ for contact force

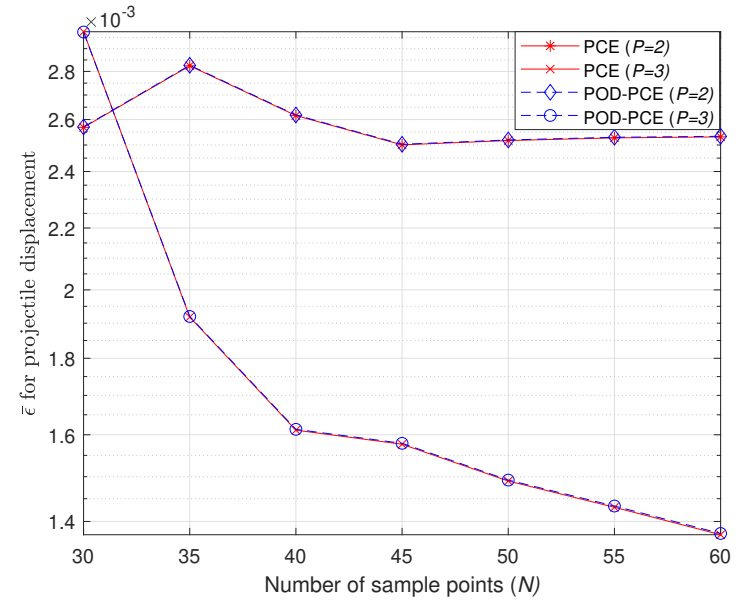

(b) $\bar{\varepsilon}$ for projectile displacement

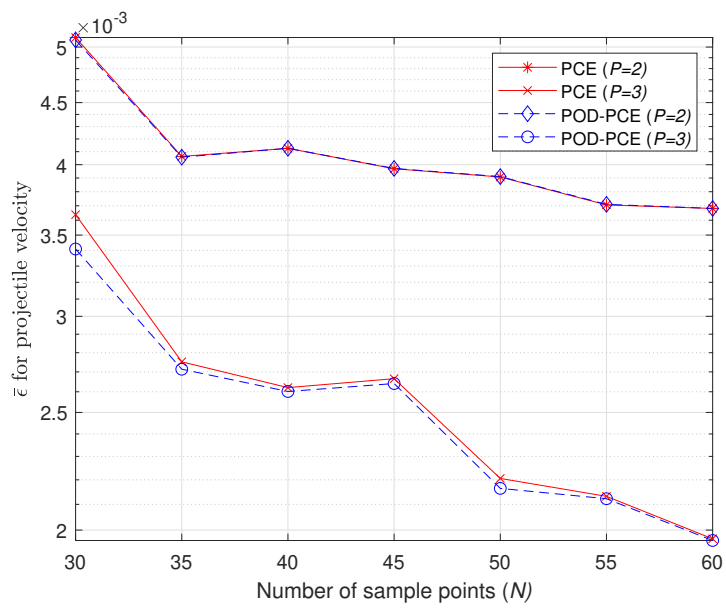

(c) $\bar{\varepsilon}$ for projectile velocity

Fig. 6: Evaluation of mean relative error for all the stochastic response quantities of the multiple impact oscillator

Table 4: Accuracy of different surrogate models in assessing the stochastic responses for the multiple impact oscillator using $N=50$

\begin{tabular}{cc|ccc|ccc|ccc}
\hline & & \multicolumn{3}{|c|}{ Contact force } & \multicolumn{3}{c|}{ Projectile displacement } & \multicolumn{3}{c}{ Projectile velocity } \\
\hline Method & $P$ & $n_{b}$ & $n_{\text {tot }}$ & $\bar{\varepsilon}$ & $n_{b}$ & $n_{\text {tot }}$ & $\bar{\varepsilon}$ & $n_{b}$ & $n_{\text {tot }}$ & $\bar{\varepsilon}$ \\
\hline PCE & 2 & - & 30010 & $1.14 \times 10^{-1}$ & - & 30010 & $2.52 \times 10^{-3}$ & - & 30010 & $3.91 \times 10^{-3}$ \\
PCE & 3 & - & 60020 & $1.05 \times 10^{-1}$ & - & 60020 & $1.49 \times 10^{-3}$ & - & 60020 & $2.21 \times 10^{-3}$ \\
POD-PCE & 2 & 31 & 310 & $1.13 \times 10^{-1}$ & 3 & 30 & $2.52 \times 10^{-3}$ & 9 & 90 & $3.91 \times 10^{-3}$ \\
POD-PCE & 3 & 31 & 620 & $1.05 \times 10^{-1}$ & 3 & 60 & $1.49 \times 10^{-3}$ & 9 & 180 & $2.16 \times 10^{-3}$ \\
\hline
\end{tabular}

exactly the same mean relative errors, however, the PCE coefficient vector was computed 3 times instead of 3001 for the POD-PCE model: it is the main advantage of the POD-PCE model.

UQ was performed on the projectile velocity also. The stochastic response behavior in time domain is shown in Figure 7e and Figure 7f: the standard deviation is shown only for the contact duration and the standard deviation is constant beyond this region. A highly non-smooth behavior is noticed in the stochastic behavior of $\dot{x}_{p}$. The peaks in the standard deviation occurred during the contacts. Such highly non-smooth moments were predicted quite well by both the surrogates using much less model evaluations as compared to MCS. The time-dependent errors for both the statistical moments are plotted in Figure 8e and Figure 8f. the errors are plotted in the contact region and the error is constant beyond this region. It is seen 


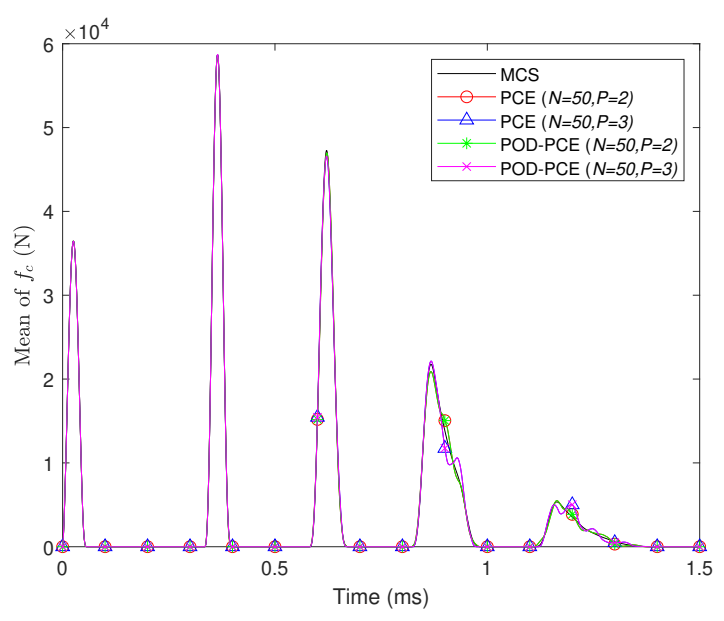

(a) Mean of $f_{c}$ (zoom on $\mathrm{x}$ axis)

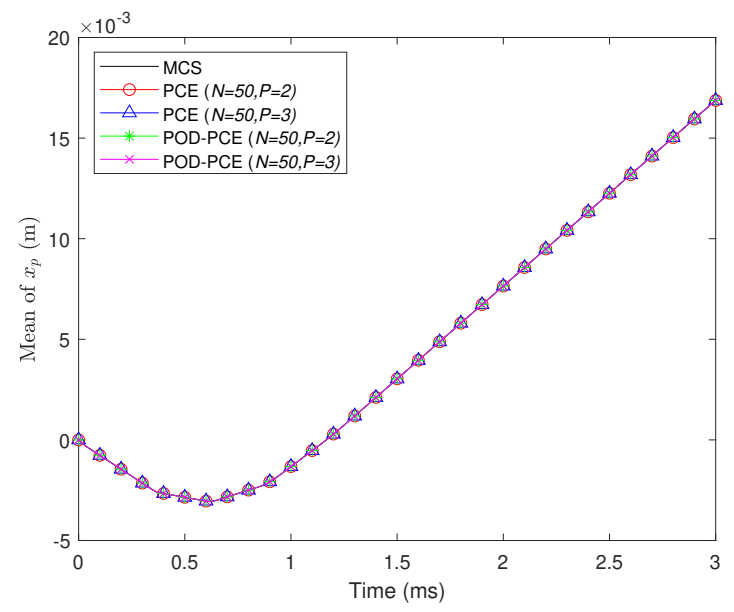

(c) Mean of $x_{p}$

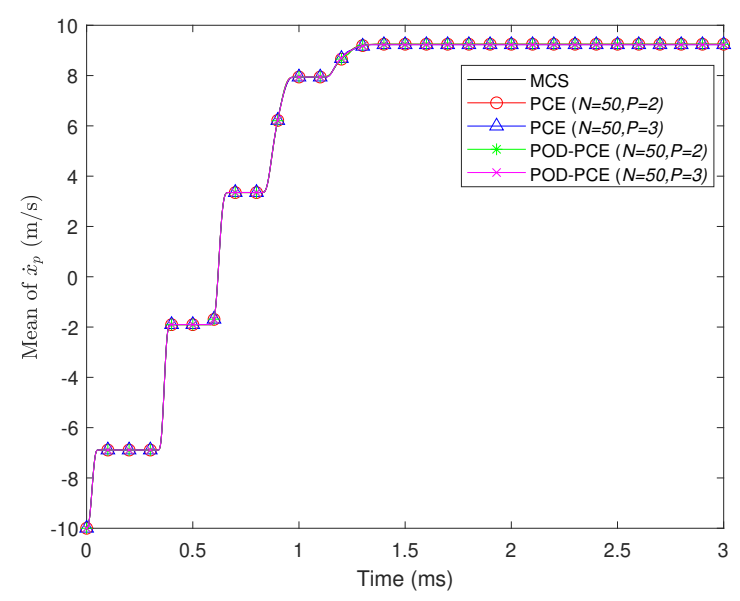

(e) Mean of $\dot{x}_{p}$

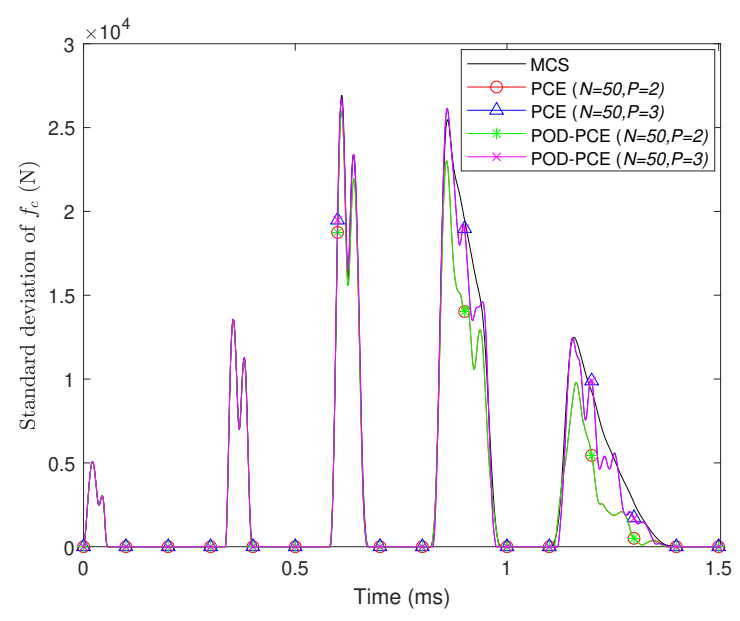

(b) Standard deviation of $f_{c}$ (zoom on $\mathrm{x}$ axis)

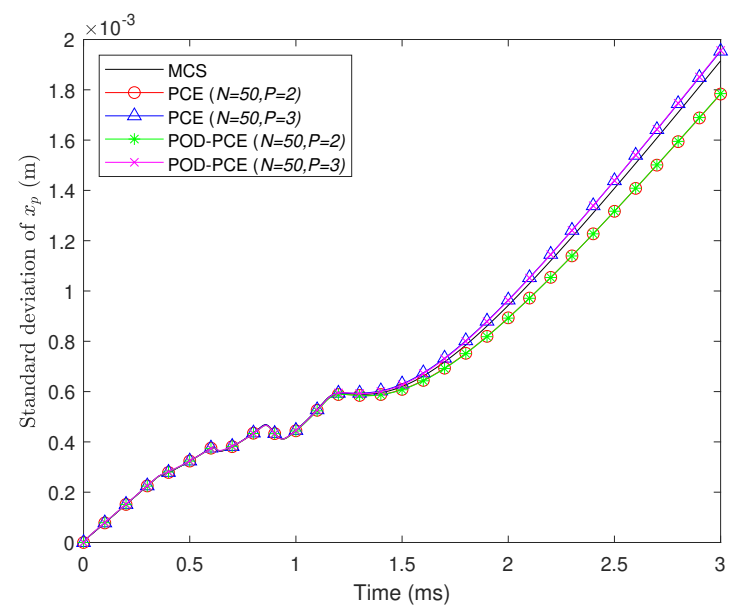

(d) Standard deviation of $x_{p}$

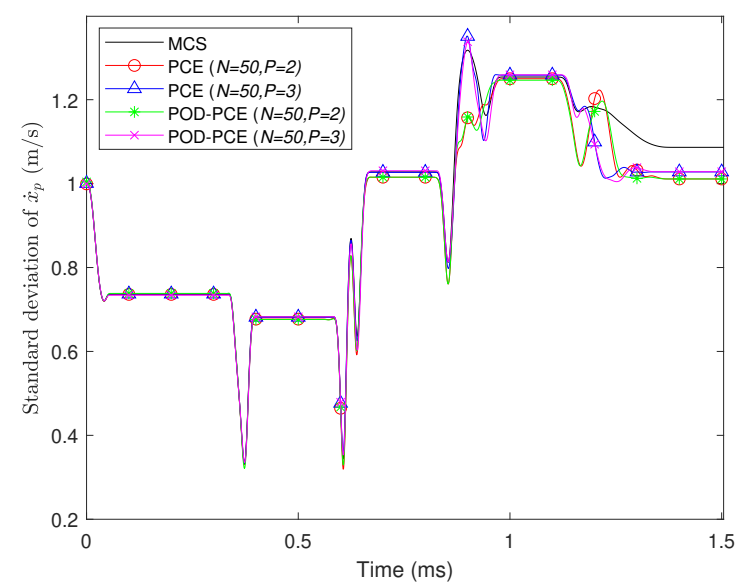

(f) Standard deviation of $\dot{x}_{p}$ (zoom on $\mathrm{x}$ axis)

Fig. 7: Time-dependent statistical moments of the stochastic responses for the multiple impact oscillator: MCS was performed using $N_{\mathrm{MCS}}=10^{4}$ model evaluations

that the magnitude of error is significantly higher for $\sigma_{\dot{x}_{p}}$ as compared to $\mu_{\dot{x}_{p}}$. Low errors are noticed using $P=3$ for both the surrogate models during the contacts. The overall accuracies are given in Table 4. It is noticeable that almost the same overall accuracies have been achieved by both the surrogates. However, for the POD-PCE model, the PCE coefficient vector was computed only 9 times: it is 3001 for the PCE model. 


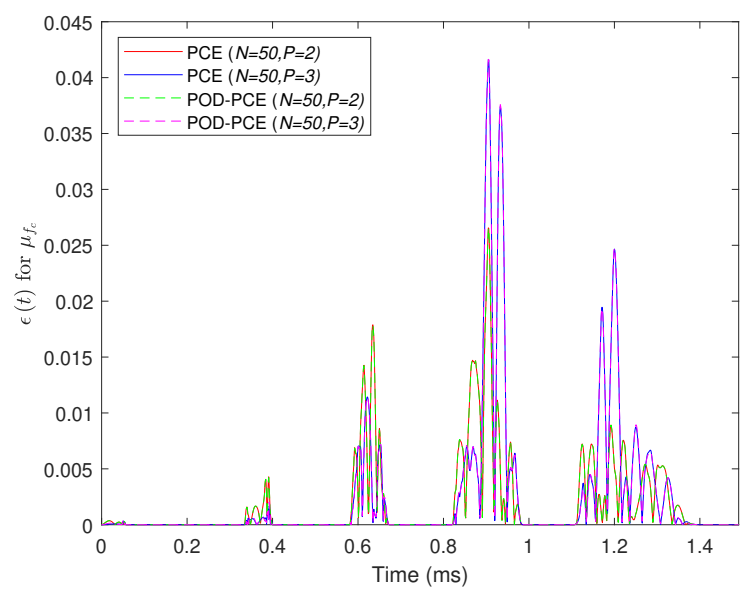

(a) Error for $\mu$ of $f_{c}$ (zoom on $\mathrm{x}$ axis)

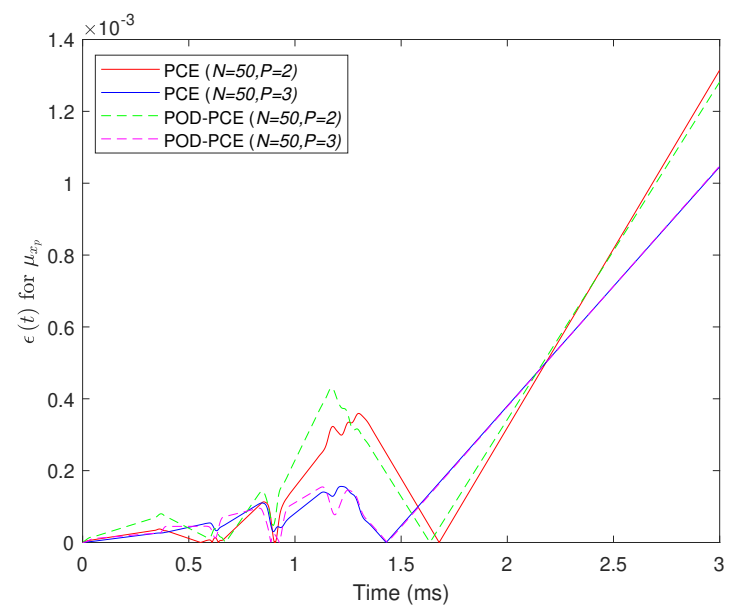

(c) Error for $\mu$ of $x_{p}$

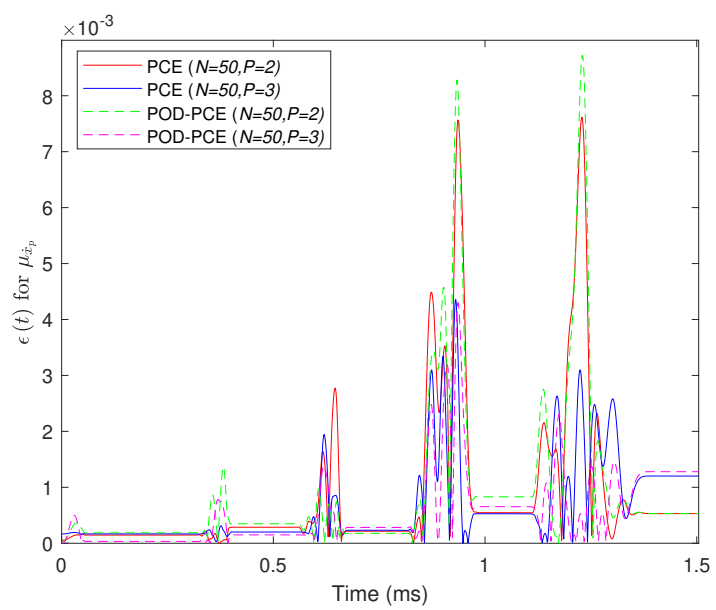

(e) Error for $\mu$ of $\dot{x}_{p}$ (zoom on $\mathrm{x}$ axis)

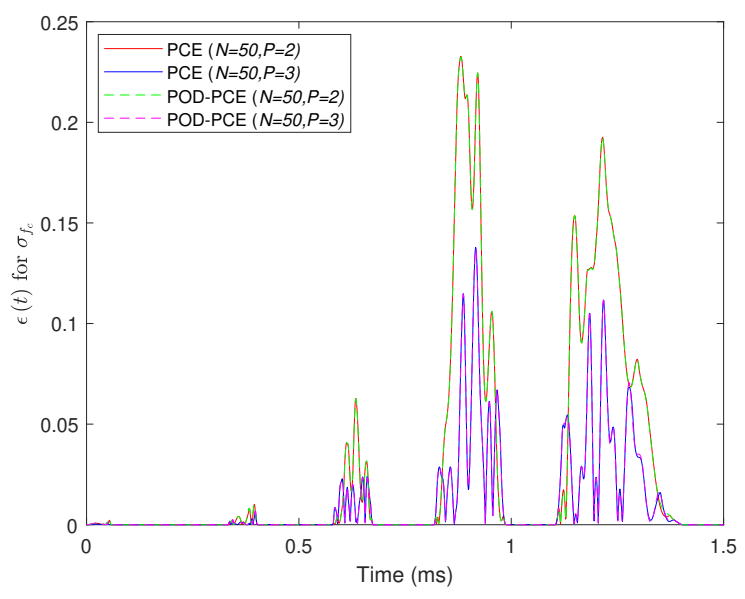

(b) Error for $\sigma$ of $f_{c}$ (zoom on $\mathrm{x}$ axis)

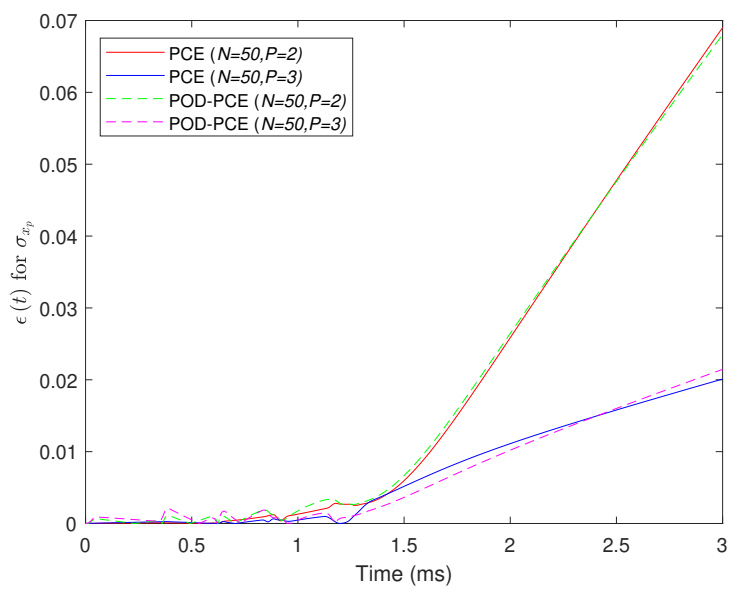

(d) Error for $\sigma$ of $x_{p}$

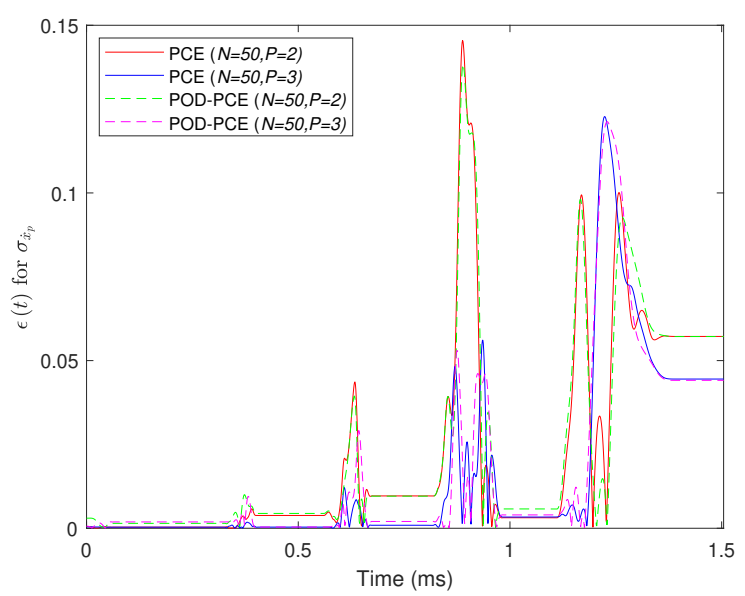

(f) Error for $\sigma$ of $\dot{x}_{p}$ (zoom on $\mathrm{x}$ axis)

Fig. 8: Time-dependent errors for the statistical moments of the response quantities of multiple impact oscillator

In a similar way to the single impact oscillator, the predicted stochastic responses by the surrogates were plotted and compared with the MCS results at the same 5 samples. The comparison is shown in Figure 9 . It seems that the contact force is predicted quite well during the first two contacts, while it is very difficult to predict the contact forces towards the end of the contacts. $\bar{\varepsilon}$ for these 5 samples was found to be the same as the $\bar{\varepsilon}$ for the $10^{4}$ samples. It is noticeable that some negative forces are predicted towards end contacts which are not physically correct. To mitigate this issue, we tried 
to identify the POD-PCE model and the PCE model by increasing the samples and the polynomial degree to $N=1000$ and $P=15$, respectively. Although it was possible to reduce the magnitude of the negative forces to some extent using such large number of samples, some negative forces were always predicted using the surrogate models. The negative forces are not a consequence of the POD but of the PCE as the PCE predicted results follow the POD-PCE predicted results. Indeed predicting a non-smooth function (i.e. contact force) by a smooth function (i.e. PCE model) is always a challenging task. On the other hand, the projectile displacement and the projectile velocity are predicted quite well at the 5 samples using both the approaches due to less non-smooth behavior than the contact force.

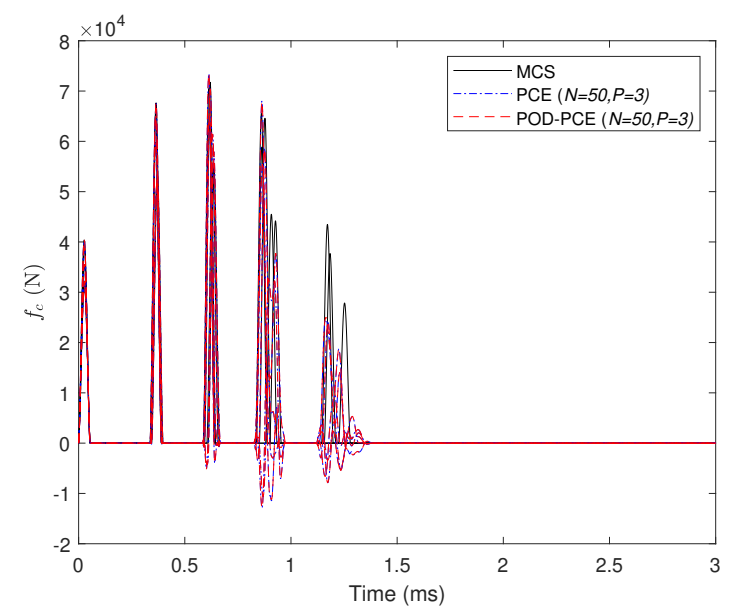

(a) Contact force

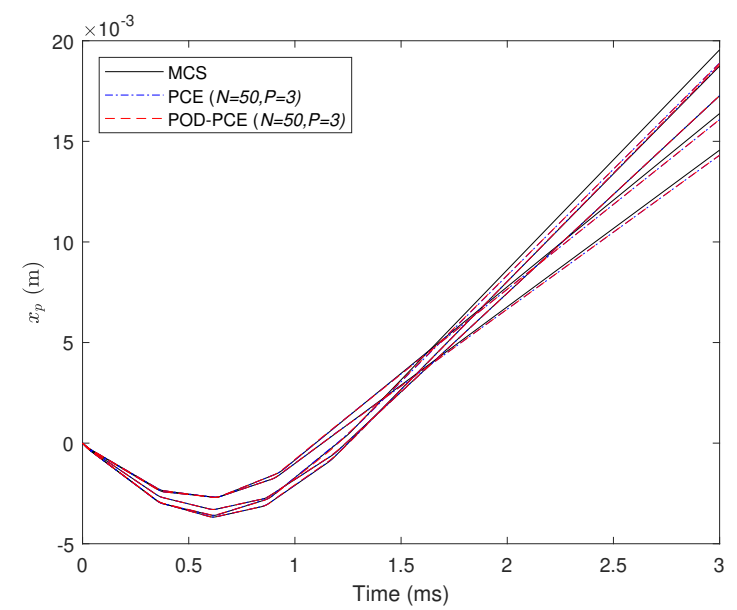

(b) Projectile displacement

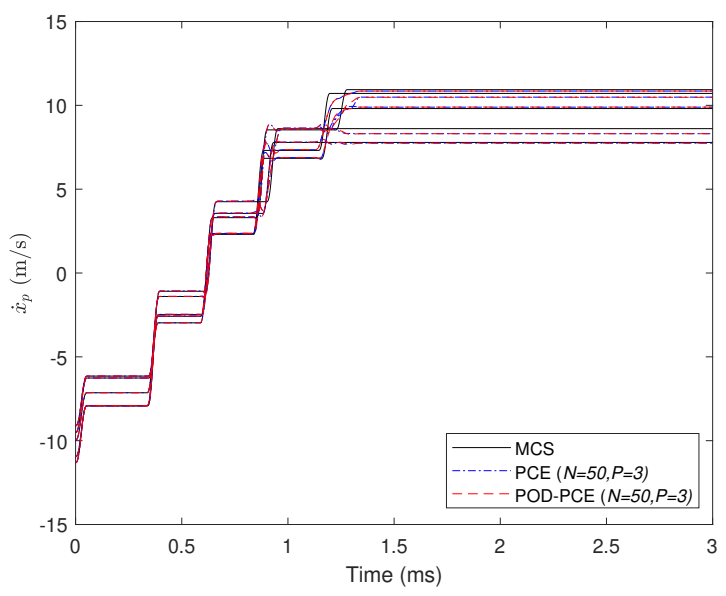

(c) Projectile velocity

Fig. 9: Comparison of responses for the multiple impact oscillator at 5 samples: $m_{p} \in$ $\{342.71,292.84,365.62,310.35,373.82\} \mathrm{g}, \quad k_{c} \in\{13.97,13.87,14.61,14.23,13.63\} \times 10^{3} \mathrm{MNm}^{-3 / 2}, \quad v_{0} \in$ $-\{11.32,9.51,9.96,9.11,10.95\} \mathrm{ms}^{-1}$

The accuracies of all the surrogate models have already been shown in Table 4 The efficiency metrics for the responses of the multiple impact oscillator is presented in Table 5. The CPU times were measured by averaging on 10 simulations. It is noticeable that the required CPU times for both surrogate models are quite low as compared to the MCS. The number of sample points is quite small as compared to the number of time-steps. As a result, the required CPU time to obtain the POD modes was approximately $0.008 \mathrm{~s}$. The CPU times for the POD-PCE models are almost 16 times and 10000 times lower than that for the PCE models and for the MCS approach, respectively. Therefore, the POD-PCE model is quite efficient as compared to the other approaches. 
Table 5: Efficiency of different approaches for the multiple impact oscillator

\begin{tabular}{ccc|ccc}
\hline & & & Contact force & Projectile displacement & Projectile velocity \\
\hline Method & $N$ & $P$ & & CPU time (s) & \\
\hline PCE & 50 & 3 & 0.181 & 0.180 & 0.181 \\
POD-PCE & 50 & 3 & 0.014 & 0.010 & 0.014 \\
\hline MCS & $10^{4}$ & - & 104.950 & 104.950 & 104.950 \\
\hline
\end{tabular}

\section{Conclusion}

A stochastic impact oscillator has been investigated in this paper by POD-PCE model. The contact between the structure and the projectile was modeled using the Hertz's law. The responses of projectile were considered for UQ. The timedependent UQ can be performed using the conventional PCE surrogate model. However, the PCE coefficient vector has to be computed at each time-step. For that reason, the time-dependent non-smooth behavior and the randomness in the model were decoupled using the proposed POD-PCE surrogate model. The time-dependent non-smooth behavior was modeled using the POD modes and the randomness in the model was propagated using the PCE model.

A nonlinear impact oscillator has been investigated for UQ with two different conditions: single contact between the structure and the projectile, multiple contacts between the structure and the projectile. UQ was performed for projectile displacement, projectile velocity and contact force. The stochastic responses were computed very well by the PCE and the POD-PCE models. Effectively, the accuracy of the predicted mean was much high as compared to the predicted standard deviation by both the surrogate models. However, non-physical contact forces were predicted for the multiple impact oscillator by both the surrogate models at some sample points due to the numerical issue with the PCE model which is the main limitation of the proposed approach. For the PCE model, the PCE coefficient vector was identified at each time-step, whereas the stochastic responses were computed using much less POD modes by the POD-PCE model which leads to computing significantly less number of PCE coefficient vector. Hence, the number of PCE model identification was much less for the POD-PCE model without losing the accuracy. As a result, the computational cost for the POD-PCE model was much lower than the other approaches. For a high-dimensional problem, obtaining the POD modes may be inefficient by the POD approach. In that case, the Krylov subspace [35] would be a good alternative to approximate the reduced modes efficiently. Further, the number of PCE coefficients could be reduced by using the sparse PCE model [36] in conjunction with the POD approach.

\section{References}

[1] Pashah, S., Massenzio, M., and Jacquelin, E., 2008. "Prediction of structural response for low velocity impact". International Journal of Impact Engineering, 35(2), feb, pp. 119-132.

[2] Apetre, N., Sankar, B., and Ambur, D., 2006. "Low-velocity impact response of sandwich beams with functionally graded core". International Journal of Solids and Structures, 43(9), may, pp. 2479-2496.

[3] Abrate, S., 2001. "Modeling of impacts on composite structures". Composite Structures, 51(2), feb, pp. 129-138.

[4] Wu, C., and Sun, C., 1996. "Low velocity impact damage in composite sandwich beams". Composite Structures, 34(1), jan, pp. 21-27.

[5] Pashah, S., Massenzio, M., and Jacquelin, E., 2008. "Structural response of impacted structure described through anti-oscillators". International Journal of Impact Engineering, 35(6), jun, pp. 471-486.

[6] Stronge, W. J., 2000. Impact mechanics. Cambridge University Press.

[7] Lucor, D., and Karniadakis, G. E., 2004. "Adaptive Generalized Polynomial Chaos for Nonlinear Random Oscillators". SIAM Journal on Scientific Computing, 26(2), pp. 720-735.

[8] Maitre, O. P. L., Mathelin, L., Knio, O., and Hussaini, M., 2010. "Asynchronous Time Integration for Polynomial Chaos Expansion of Uncertain Periodic Dynamics". Discrete Continuum Dynamic Systems - Series A, 28(1), pp. 199226.

[9] Gerritsma, M., Steen, J.-B. V. D., Vos, P., and Karniadakis, G., 2010. "Time-dependent generalized polynomial chaos". Journal of Computational Physics, 229(22), pp. 8333-8363.

[10] Spiridonakos, M., and Chatzi, E., 2015. "Metamodeling of dynamic nonlinear structural systems through polynomial chaos NARX models". Computers \& Structures, 157, pp. 99-113.

[11] Wan, X., and Karniadakis, G. E., 2005. "An adaptive multi-element generalized polynomial chaos method for stochastic differential equations". Journal of Computational Physics, 209(2), pp. 617-642.

[12] Sarrouy, E., Dessombz, O., and Sinou, J. J., 2012. "Stochastic analysis of the eigenvalue problem for mechanical 
systems using polynomial chaos expansion-application to a finite element rotor". Journal of Vibration and Acoustics, Transactions of the ASME, 134(5).

[13] Nechak, L., Berger, S., and Aubry, E., 2012. "Prediction of random self friction-induced vibrations in uncertain dry friction systems using a multi-element generalized polynomial chaos approach". Journal of Vibration and Acoustics, Transactions of the ASME, 134(4).

[14] Bhusal, R., and Subbarao, K., 2019. "Uncertainty Quantification Using Generalized Polynomial Chaos Expansion for Nonlinear Dynamical Systems With Mixed State and Parameter Uncertainties”. Journal of Computational and Nonlinear Dynamics, 14(2), feb.

[15] Luchtenburg, D. M., Brunton, S. L., and Rowley, C. W., 2014. "Long-time uncertainty propagation using generalized polynomial chaos and flow map composition". Journal of Computational Physics, 274, pp. 783-802.

[16] Mai, C. V., and Sudret, B., 2017. "Surrogate models for oscillatory systems using sparse polynomial chaos expansions and stochastic time warping". SIAM/ASA Journal on Uncertainty Quantification, 5(1), pp. 540-571.

[17] Bhattacharyya, B., Jacquelin, E., and Brizard, D., 2020. "A Kriging-NARX model for uncertainty quantification of nonlinear stochastic dynamical systems in time domain”. Journal of Engineering Mechanics, 146(7), pp. 1-21.

[18] Chinesta, F., Ladeveze, P., and Cueto, E., 2011. “A Short Review on Model Order Reduction Based on Proper Generalized Decomposition”. Archives of Computational Methods in Engineering, 18(4), nov, pp. 395-404.

[19] Chatterjee, A., 2000. "An introduction to the proper orthogonal decomposition". Current Science, 78(7), pp. 808-817.

[20] Kunisch, K., and Volkwein, S., 2002. "Galerkin Proper Orthogonal Decomposition Methods for a General Equation in Fluid Dynamics”. SIAM Journal on Numerical Analysis, 40(2), jan, pp. 492-515.

[21] Schmid, P. J., 2010. "Dynamic mode decomposition of numerical and experimental data". Journal of Fluid Mechanics, 656, pp. 5-28.

[22] Blatman, G., and Sudret, B., 2013. "Sparse polynomial chaos expansions of vector-valued response quantities". In ICOSSAR, June 16-20, New York, USA.

[23] Moore, B., 1979. "Principal component analysis in nonlinear systems: Preliminary results". In 18th IEEE Conference on Decision and Control including the Symposium on Adaptive Processes, Dec. 12-14, Fort Lauderdale, FL, USA, IEEE, pp. 1057-1060.

[24] Kunisch, K., and Volkwein, S., 1999. "Control of the Burgers Equation by a Reduced-Order Approach Using Proper Orthogonal Decomposition”. Journal of Optimization Theory and Applications, 102(2), aug, pp. 345-371.

[25] Higdon, D., Gattiker, J., Williams, B., and Rightley, M., 2008. "Computer Model Calibration Using High-Dimensional Output”. Journal of the American Statistical Association, 103(482), pp. 570-583.

[26] Xiu, D., and Karniadakis, G. E., 2002. "The Wiener-Askey polynomial chaos for stochastic differential equation". SIAM Journal on Scientific Computing Scientific Computing, 24(2), pp. 619-644.

[27] Jacquelin, E., Baldanzini, N., Bhattacharyya, B., Brizard, D., and Pierini, M., 2019. "Random dynamical system in time domain: A POD-PC model". Mechanical Systems and Signal Processing, 133, nov, p. 106251.

[28] Bhattacharyya, B., 2018. “A Critical Appraisal of Design of Experiments for Uncertainty Quantification”. Archives of Computational Methods in Engineering, 25(3), pp. 727-751.

[29] Sirovich, L., 1987. "Turbulence and the dynamics of coherent structures Part I: Coherent structures". Quarterly of Applied Mathematics, 45(3), pp. 561-571.

[30] Berkooz, G., Holmes, P., and Lumley, J. L., 1993. "The Proper Orthogonal Decomposition in the Analysis of Turbulent Flows”. Annual Review of Fluid Mechanics, 25(1), jan, pp. 539-575.

[31] Azeez, M., and Vakakis, A., 2001. "Proper orthogonal decomposition (POD) of a class of vibro-impact oscillations". Journal of Sound and Vibration, 240(5), mar, pp. 859-889.

[32] Mai, C. V., Spiridonakos, M. D., Chatzi, E. N., and Sudret, B., 2016. "Surrogate modeling for stochastic dynamical systems by combining nonlinear autoregressive with exogenous input models and polynomial chaos expansions". International Journal for Uncertainty Quantification, 6(4), pp. 313-339.

[33] Johnson, K. L., 1985. Contact Mechanics. Cambridge University Press.

[34] Buezas, F. S., Rosales, M. B., and Sampaio, R., 2013. "Propagation of uncertainties and multimodality in the impact problem of two elastic bodies". International Journal of Mechanical Sciences, 75, oct, pp. 145-155.

[35] Freund, R. W., 2000. "Krylov-subspace methods for reduced-order modeling in circuit simulation". Journal of Computational and Applied Mathematics, 123(1-2), pp. 395-421.

[36] Blatman, G., and Sudret, B., 2011. “Adaptive sparse polynomial chaos expansion based on least angle regression". Journal of Computational Physics, 230(6), pp. 2345-2367. 


\section{List of Figures}

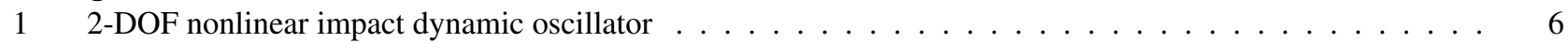

\begin{tabular}{|llll}
\hline 2 & Evaluation of the mean relative errors of all the stochastic response quantities for the single impact oscillator & 7
\end{tabular}

3 Time-dependent statistical moments of the stochastic response quantities for the single impact oscillator: MCS was performed using $N_{\mathrm{MCS}}=10^{4}$ model evaluations $\ldots \ldots \ldots \ldots \ldots$

4 Time-dependent errors for the statistical moments of the stochastic responses of single impact oscillator . . 9

$5 \quad$ Comparison of responses for single impact oscillator at 5 samples: $m_{p} \in\{342.71,292.84,365.62,310.35,373.82\} \mathrm{g}$, $k_{c} \in\{13.97,13.87,14.61,14.23,13.63\} \times 10^{3} \mathrm{MN} \mathrm{m}^{-3 / 2}, v_{0} \in-\{11.32,9.51,9.96,9.11,10.95\} \mathrm{m} \mathrm{s}^{-1} \mid \ldots .11$

$6 \quad$ Evaluation of mean relative error for all the stochastic response quantities of the multiple impact oscillator . 12

7 Time-dependent statistical moments of the stochastic responses for the multiple impact oscillator: MCS was

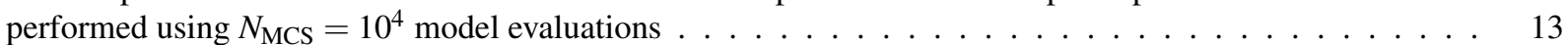

8 Time-dependent errors for the statistical moments of the response quantities of multiple impact oscillator $\quad 14$

9 Comparison of responses for the multiple impact oscillator at 5 samples: $m_{p} \in\{342.71,292.84,365.62,310.35,373.82\} \mathrm{g}$, $k_{c} \in\{13.97,13.87,14.61,14.23,13.63\} \times 10^{3} \mathrm{MN} \mathrm{m}^{-3 / 2}, v_{0} \in-\{11.32,9.51,9.96,9.11,10.95\} \mathrm{m} \mathrm{s}^{-1} \ldots \ldots$ 


\section{List of Tables}

$1 \quad$ Parameters of the input random variables for the impact dynamic oscillator $\ldots \ldots \ldots \ldots$

2 Accuracy of different surrogate models in assessing the stochastic response quantities for the single impact

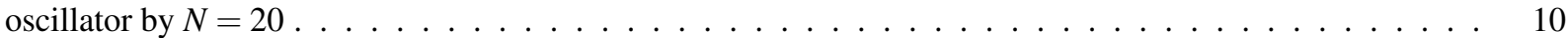

$3 \quad$ Efficiency of different approaches for the single impact oscillator $\ldots \ldots \ldots \ldots \ldots \ldots$

4 Accuracy of different surrogate models in assessing the stochastic responses for the multiple impact oscillator

5 Efficiency of different approaches for the multiple impact oscillator $\ldots \ldots \ldots \ldots \ldots$ 